\title{
Predicting the Noise of an Open Rotor in a Wind Tunnel
}

\author{
P. Sureshkumar, M. J. Kingan and A. B. Parry
}

October 29, 2018

\section{Abstract}

A study of the impact of reverberation on cruise regime acoustic testing of contra-rotating open rotors in a wind tunnel is presented. Models for the rotor tone noise generated in closed cross section wind tunnels are derived and used in the analysis. The models account for rectangular and circular cross sections, rigid or impedance boundaries and, for the rectangular wind tunnel case, the rotor axis may be arbitrarily located within the tunnel (but aligned with the flow direction). These models are used to generate simple simulations of model-scale open rotor testing in two real wind tunnels suited to high speed rotor testing - the Transonic Wind Tunnel in Bedford, UK and the S1MA wind tunnel in Modane, France. In the analysis, the effect of various modelling parameters on the reverberant noise field is investigated. It is shown that open rotor interaction tone noise may be particularly susceptible to the impact of reverberation and that for many tones the reverberant field may persist and contribute to a contaminated noise field, even for wind tunnels with acoustic treatments on the walls of the working section.

\section{Introduction}

Open rotor aero-engines promise a potentially significant reduction in fuel burn when compared to current, conventional aero-engines. However, minimisation of the noise from these engines is important if they are to become viable aircraft propulsors. A cut-away view of a typical open rotor design is shown in Figure 1.

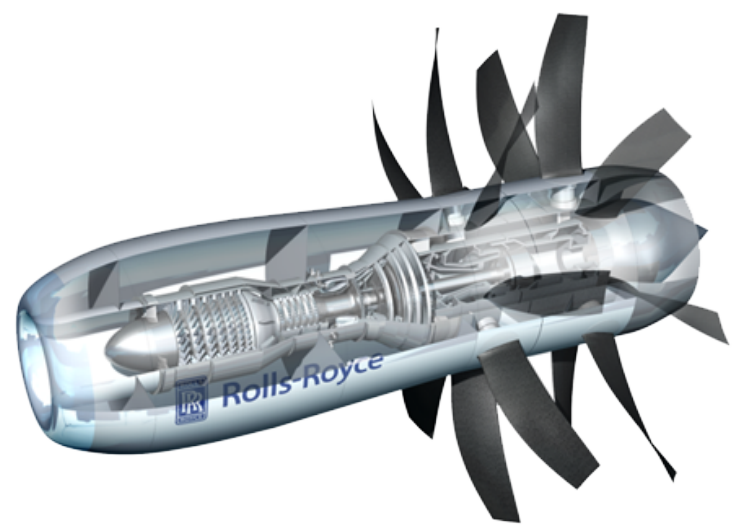

Figure 1: Cut-away view of an advanced open rotor. Picture courtesy of Rolls-Royce plc.

Over the last 30 years, Rolls-Royce has been involved in several experimental campaigns to assess the cruise performance and noise of open rotors. Measurements of the noise generated by scale open rotor test rigs immersed in high speed flows (representative of cruise flight conditions) are used to estimate cabin noise and en-route noise levels. Corrections must be applied to the model-scale rig test data in order to 
estimate the noise levels produced by a full-scale engine. These corrections are used to compensate for scale factor, variations in ambient pressure and temperature, background noise during testing and contamination due to the reverberation. The focus of this paper is to use theoretical models to analyse the impact of tunnel reverberation and draw conclusions on the suitability of acoustic testing in a closed test section wind tunnel.

Kirker ${ }^{1}$ describes the testing of Rolls-Royce's Rig 140 (a model scale open rotor test rig) in the Aircraft Research Association's (ARA) Transonic Wind Tunnel. This tunnel has a rectangular cross section and rigid walls. Wood and Newman ${ }^{2}$ describe the development and implementation of a removable acoustic liner designed specifically for this tunnel's test section. The purpose of this liner was to reduce the effect of reverberation in the test section over a range of frequencies. The liner was optimised for Rolls-Royce's propeller testing requirements at the time. Installing the liner meant that acoustic measurements could be more readily undertaken. Therefore, during acoustic testing, the liner was installed in the test-section of the wind tunnel. Commissioning tests showed that the liner was only effective for flow Mach numbers below approximately 0.6 and a degradation in liner performance was noted at higher wind tunnel flow speeds. Parry et al. ${ }^{3}$ later describe the high-speed testing of Rolls-Royce's refurbished model scale open rotor test rig, Rig 145, in the ARA's Transonic Wind Tunnel. Similarly, the noise measurements from these tests are suspected to be contaminated by reverberation for the cases simulating cruise conditions, despite the use of the acoustic liner. A photograph of the tunnel test section during these tests is presented in Figure 2. In their paper a significant number of experimental acoustic measurements are presented but because of concerns over reverberation effects in the tunnel, these were confined to axial positions close to the rotor, where the effect seemed less significant.

Theoretical models for calculating the sound pressure produced by an open rotor in a wind tunnel are presented. Wind tunnels that might typically be used for high speed open rotor testing are modelled in order to assess the impact of reverberation in these tunnel setups. Understanding the effect would allow the industrial partner, Rolls-Royce, to improve the accuracy of their estimates for Open Rotor cruise noise. Only the tonal noise component of Open Rotors is considered here and the frequency domain methods popularised by Hanson ${ }^{4-6}$ are the basis for rotor tone noise prediction.

The analysis used in this paper makes use of an analytic Green's function to account for the reflection caused by the wind tunnel walls. Deriving Green's functions for ducts with internal flows, rotating sources and acoustic treatments at the boundary has been the subject of much published research. A number of these papers ${ }^{7-9}$ were motivated by investigations of noise inside a turbofan aero-engine and therefore usually present models for circular or annular cross-sections. A similar method for noise prediction is proposed in this paper but the geometries considered reflect typical wind tunnel setups. In a wind tunnel, the cross-sectional area is large relative to the rotor disc area. In this analysis the near-field region of the source is also of significant interest. Both of these requirements means that a significant number of modes must be included in the calculation in order to achieve a converged solution and thus the computational cost is large.

In Section 3 the procedure to derive the relevant theoretical models is outlined. The wind tunnel Green's functions for a rectangular cross-section and circular cross-section wind tunnel are presented, with further derivation showing how expressions for rotor loading sources can be obtained. Graf's addition theorem is used in the derivation of the expression for a rectangular cross-section wind tunnel in Section 3.1.1, which simplifies the final expression and has the benefit of reducing the computational cost of evaluating this expression. Section 4 describes analysis of open rotor tone noise in wind tunnel environments using the proposed models. The models are used to assess and understand the impact of tunnel reverberation. A comparison is made between models for an open rotor in the free-field and a wind tunnel with an impedance boundary condition at the walls. The comparison shows how under certain conditions predictions within a wind tunnel can theoretically be similar to those made in the free-field. The differences in the acoustic field generated in rectangular and circular cross-section wind tunnels is also investigated. Finally, simulations of open rotor tone noise in two existing wind tunnel setups are presented. The analyses provide rudimentary insight into the impact that tunnel reverberation might have when testing at these facilities. The two facilities are the Transonic Wind Tunnel (TWT) at the Aircraft Research Association (Bedford, UK) and ONERA's S1MA wind tunnel (Modane, France). The former of the two has a rectangular cross-section in the test section whilst the latter is circular. 


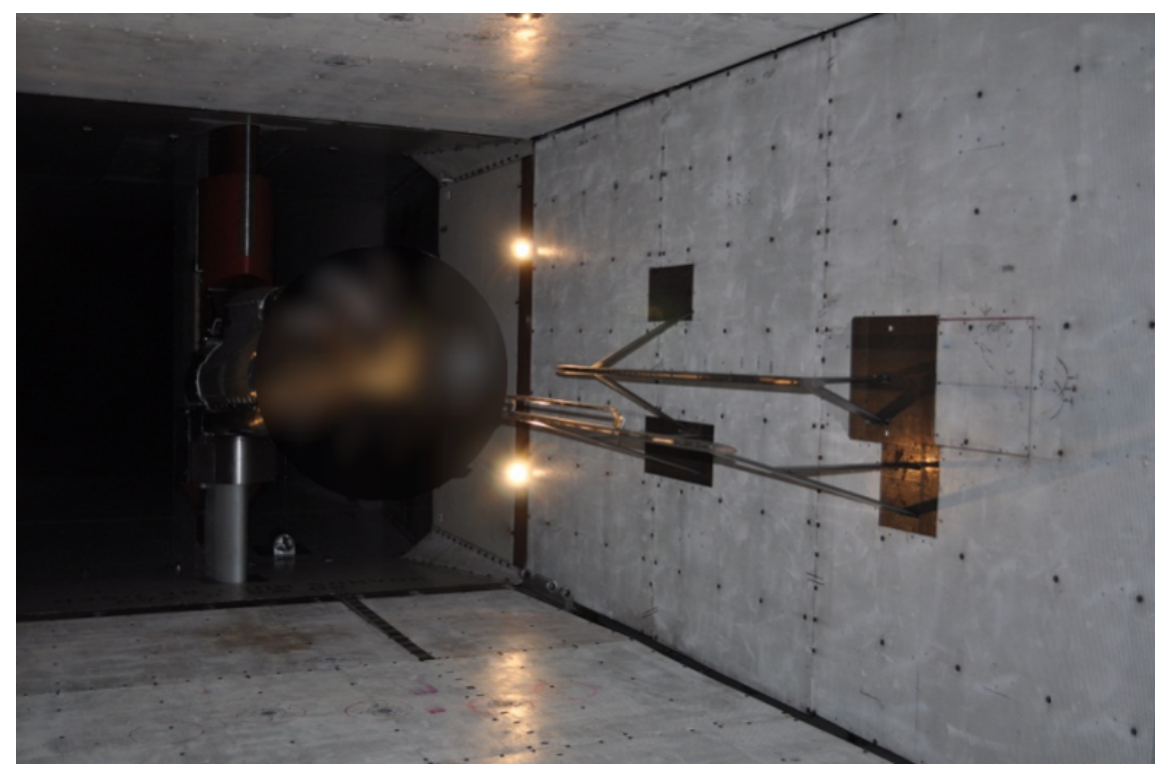

Figure 2: A photograph of model scale open rotor tests conducted at the ARA's transonic wind tunnel in Bedford, UK (rotor blurred). Noise was measured using microphones flush-mounted in the rails mounted off the wind tunnel wall, which can also be seen in the photograph.

\section{Formulation}

The wind tunnel is assumed infinitely long and so in this analysis end reflections are ignored. If the tunnel setup is sufficiently long to allow cut-off modes to decay, reflections of these modes from the ends of the wind tunnel should be insignificant. The analysis in this paper considers sufficiently high frequencies, duct radii and only purely real impedances, such that end reflections of most modes are not likely to impact the solution. Also, the tunnel's boundaries do not vary along the tunnel axis. The axial co-ordinate is denoted by the subscript 1 and is positive downstream. This analysis is also limited to the consideration of rectangular and circular cross sections. The rotational axis of the sources (i.e. rotor) and the wind tunnel centreline are parallel. In the circular cross section model the two axes are collinear, however the formulation of the rectangular cross section model allows the axis of rotation to be located anywhere within the tunnel cross section. It is also assumed that an impedance boundary condition would be sufficient to model the presence of an acoustic liner on the tunnel's boundaries.

The dominant source of noise is assumed to be produced by loading on the rotor blades. It is assumed the blade loading is purely due to interactions with the hydrodynamic field and any acoustic scattering from the rotor blades is ignored. Therefore only a formulation for dipole sources is presented. However, the method could be extended in the same manner to consider the noise due to monopole (thickness) and perhaps quadrupole sources, which are also potential sources of rotor tone noise. We note that it has been shown recently by Envia ${ }^{10}$ that quadrupole noise can be a significant component of some Open Rotor tones in the transonic flight regime. However, predicting quadrupole noise is practically difficult. First, all quadrupole sources must be predicted, typically using a high-fidelity CFD method. Next the radiated acoustic field is calculated using a method such as the Ffowcs-Williams Hawkings equation. For quadrupole sources this requires an integration over the volume enclosing the significant quadrupole sources surrounding the blades (which requires an objective and robust criterion for estimating the extent of this volume). The evaluation of this integral for free-field radiation is computationally expensive and Envia ${ }^{10}$ has developed an efficient method for this purpose, based on a high-blade-count asymptotic approximation. It is unlikely that such an approach could be adopted in the method we propose here which would likely be computationally very intensive for calculating quadrupole noise.

The tunnel mean flow velocity is constant and uniform across the tunnel cross section. This is a crude 
assumption, especially since it is thought that at high speed the flow may modify the impedance (and thus 'performance') of the tunnel liner. However, the plug flow assumption makes the problem more tractable. It is thought that the wavelengths of the tone harmonics of interest may be larger than the thickness of the tunnel wall boundary layers but this cannot be confirmed without measurement of the tunnel wall boundary layer. In general, the boundary layer is typically thin in the test section of a high speed wind tunnel and often boundary layer suction is applied at the start of the test section. In such cases it should be plausible to ignore acoustic refraction in the wall boundary layer. Finally, the effects of flow distortions induced by the open rotor itself and acoustic scattering from any other structures in the tunnel (such as the open rotor's centrebody) are ignored. In theory such effects could have a considerable effect on the noise field. For example, in the free-field, centrebody scattering has been shown to add a significant contribution to the level of rotor-rotor interaction tones of an open rotor ${ }^{11}$. In a wind tunnel, it is likely that the centrebody, as an extra scattering surface, would materially change the acoustic field.

The flow is assumed to be isentropic, therefore $p=\rho c_{0}^{2}$ where $p$ denotes the acoustic pressure perturbation, $\rho$ is the corresponding density perturbation and $c_{0}$ is the speed of sound in the ambient fluid. Introducing the isentropic assumption into eq. 4.13 from Goldstein ${ }^{12}$ gives the following expression, which we use to determine the unsteady pressure at location $\mathbf{x}=\left\{x_{1}, x_{2}, x_{3}\right\}$ and time $t$ produced by the loading exerted by the blades of the open rotor on the surrounding fluid.

$$
p(\mathbf{x}, t)=\int_{-T}^{T} \int_{S_{b}(\tau)} f_{i}(\mathbf{y}, \tau) \frac{\partial}{\partial y_{i}} g(\mathbf{y}, \tau \mid \mathbf{x}, t) \mathrm{d} S(\mathbf{y}) \mathrm{d} \tau .
$$

Note that $-T<\tau<T$ is the interval of time over which sound is emitted and is assumed to be infinitely large. $g$ is a Green's function which satisfies the convected wave equation

$$
\left[\frac{\partial^{2}}{\partial y_{1}^{2}}+\frac{\partial^{2}}{\partial y_{2}^{2}}+\frac{\partial^{2}}{\partial y_{3}^{2}}-\frac{1}{c_{0}^{2}}\left(\frac{\partial}{\partial \tau}+U \frac{\partial}{\partial y_{1}}\right)^{2}\right] g(\mathbf{y}, \tau \mid \mathbf{x}, t)=-\delta(t-\tau) \delta(\mathbf{y}-\mathbf{x}),
$$

where $U$ is the mean flow velocity in the wind tunnel and is aligned with the tunnel's axial co-ordinate. $f_{i}$ is the force per unit area exerted by the blades on the air, which is assumed to be dominated by the lift force. We choose Green's functions that simulate the following two conditions; either a wind tunnel with rigid walls and purely axial flow, or a wind tunnel with acoustically lined walls and no flow. Either condition will give rise to a different boundary condition which the Green's function must also satisfy and these are provided at the relevant point in the paper. A wind tunnel with acoustically lined walls and flow is more complicated to consider because of the requirement to calculate an additional set of 'surface' modes. Due to the difficulty in accurately predicting these extra modes, the case of flow in a wind tunnel with lined walls is not considered in this paper.

The blades are modelled as being infinitely thin so that the integration over the blade surface $S_{b}$ can be replaced by an integration over the blade planform area and $f_{i}$ replaced by $L^{(b)} n_{i}$ where $L^{(b)}$ is the lift force per unit area on the $b^{\text {th }}$ blade and $n_{i}$ is now a unit vector in the direction of the local 'lift' force exerted by the blades on the fluid. It will be more convenient to work in cylindrical coordinates $\left\{y_{1}, r, \phi\right\}$ where $r$ and $\phi$ are co-ordinates for the radius and azimuthal angle.

Also, $n_{i} \frac{\partial}{\partial y_{i}}$ can be written in cylindrical coordinates as

$$
n_{i} \frac{\partial}{\partial y_{i}}=n_{1} \frac{\partial}{\partial y_{1}}+n_{\phi} \frac{1}{r} \frac{\partial}{\partial \phi}+n_{r} \frac{\partial}{\partial r}
$$

where $n_{r}$ and $n_{\phi}$ are the radial and azimuthal components of the unit vector $\mathbf{n}$. The blades rotate at $\Omega \mathrm{rad} / \mathrm{s}$ and the lift force is assumed to be aligned normal to the local flow direction. Therefore the components of the unit vector may be defined as 


$$
n_{1}=\sin \alpha, n_{\phi}=\cos \alpha, n_{r}=0
$$

where $\tan \alpha=\Omega r / U$.

The rotor is assumed to have $B$ identical, evenly spaced blades. If $S_{p}^{(b)}$ denotes the planform area of the $b^{\text {th }}$ rotor blade, eq. (1) can be rewritten as follows.

$$
p(\mathbf{x}, t)=\sum_{b=0}^{B-1} \int_{-\infty}^{\infty} \int_{S_{p}^{(b)}(\tau)} L^{(b)}\left[\sin \alpha \frac{\partial g}{\partial y_{1}}+\frac{\cos \alpha}{r} \frac{\partial g}{\partial \phi}\right] \mathrm{d} S(\mathbf{y}) \mathrm{d} \tau
$$

It will be convenient to express the lift force per unit area on the planform of the $b^{\text {th }}$ blade in terms of its radial and chordwise blade coordinates $r$ and $X^{(b)}$ i.e. $L^{(b)}\left(X^{(b)}, r, \tau\right)$. The location of a point on the $b^{\text {th }}$ blade can be expressed in terms of the cylindrical coordinate system using the equations below.

$$
\begin{gathered}
y_{1}=\left(s+X^{(b)}\right) \cos \alpha \\
\phi=\Omega \tau-\left(s+X^{(b)}\right) \frac{\sin \alpha}{r}+\frac{2 \pi b}{B}
\end{gathered}
$$

The hub and the tip of the blades are located at radius $R_{h}$ and $R_{t}$ respectively. The chordwise coordinate $X^{(b)}$ has its origin at the mid-chord such that $-c / 2<X^{(b)}<c / 2$ where $c$ denotes the blade chord length. Note that $s$ denotes the blade 'sweep'. Eq. (5) becomes

$$
p(\mathbf{x}, t)=\sum_{b=0}^{B-1} \int_{-\infty}^{\infty} \int_{R_{h}}^{R_{t}} \int_{-c / 2}^{c / 2} L^{(b)}\left[\sin \alpha \frac{\partial g}{\partial y_{1}}+\frac{\cos \alpha}{r} \frac{\partial g}{\partial \phi}\right] \mathrm{d} X^{(b)} \mathrm{d} r \mathrm{~d} \tau .
$$

The Green's function is given by

$$
\begin{aligned}
& g(\mathbf{y}, \tau \mid \mathbf{x}, t)=\frac{-\mathrm{i}}{4 \pi} \sum_{n_{2}} \sum_{n_{3}} \int_{-\infty}^{\infty} \frac{\Psi_{n_{2}, n_{3}}\left(y_{2}, y_{3}\right) \Psi_{n_{2}, n_{3}}\left(x_{2}, x_{3}\right)}{k_{n_{2}, n_{3}} \Gamma_{n_{2}, n_{3}}} \\
& \quad \times \exp \left\{\mathrm{i} \omega(t-\tau)-\mathrm{i} \frac{M \kappa}{\beta^{2}}\left(y_{1}-x_{1}\right)-\mathrm{i} \frac{k_{n_{2}, n_{3}}}{\beta^{2}}\left|y_{1}-x_{1}\right|\right\} \mathrm{d} \omega
\end{aligned}
$$

where $\kappa=\omega / c_{0}, k_{n_{2}, n_{3}}^{2}=\kappa^{2}-\beta^{2} \varkappa_{n_{2}, n_{3}}^{2}, \beta^{2}=1-M^{2}, M=U / c_{0}$ and $\varkappa_{n_{2}, n_{3}}$ and $\Psi_{n_{2}, n_{3}}$ are an eigenvalue/eigenfunction pair which satisfy the following two-dimensional Helmholtz equation

$$
\left(\frac{\partial^{2}}{\partial y_{2}^{2}}+\frac{\partial^{2}}{\partial y_{3}^{2}}+\varkappa_{n_{2}, n_{3}}^{2}\right) \Psi_{n_{2}, n_{3}}=0
$$

as well as whichever of the boundary conditions is relevant for the case being modelled. The Green's function is transformed to the frequency domain by

$$
g(\mathbf{y}, \tau \mid \mathbf{x}, t)=\int_{-\infty}^{\infty} G_{\omega}(\mathbf{y} \mid \mathbf{x}) \frac{\exp \{\mathrm{i} \omega(t-\tau)\}}{2 \pi} \mathrm{d} \omega
$$


and so

$$
G_{\omega}(\mathbf{y} \mid \mathbf{x})=\frac{-\mathrm{i}}{2} \sum_{n_{2}} \sum_{n_{3}} \frac{\Psi_{n_{2}, n_{3}}\left(y_{2}, y_{3}\right) \Psi_{n_{2}, n_{3}}\left(x_{2}, x_{3}\right)}{\Gamma_{n_{2}, n_{3}} k_{n_{2}, n_{3}}} \exp \left\{-\mathrm{i} \frac{M \kappa}{\beta^{2}}\left(y_{1}-x_{1}\right)-\mathrm{i} \frac{k_{n_{2}, n_{3}}}{\beta^{2}}\left|y_{1}-x_{1}\right|\right\}
$$

where

$$
\Gamma_{n_{2}, n_{3}}=\int_{A} \Psi_{n_{2}, n_{3}}^{2}\left(y_{2}, y_{3}\right) \mathrm{d} A
$$

and $A$ is the cross sectional area of the wind tunnel.

Note that this frequency domain Green's function $G_{\omega}$ must also satisfy the appropriate boundary conditions. If the tunnel surfaces are rigid, $G_{\omega}$ would satisfy

$$
n_{i} \frac{\partial G_{\omega}}{\partial y_{i}}=0
$$

where $n_{i}$ is a unit vector normal to the tunnel wall surfaces, pointing out of the tunnel volume. In the frequency domain, the acoustic impedance $Z$ is defined by $Z=\tilde{p} / \tilde{u_{n}}$, where $\tilde{u_{n}}$ is the acoustic velocity normal to the wind tunnel wall and the normal is directed out of the wind tunnel volume. Defining the specific acoustic admittance $\Lambda$ as

$$
\Lambda=\frac{\rho_{0} c_{0}}{Z}=\frac{\rho_{0} c_{0} \tilde{u_{n}}}{\tilde{p}}
$$

and coupling this with the linearised Euler equation for momentum results in the following formulation of the no-flow impedance boundary condition

$$
n_{i} \frac{\partial G_{\omega}}{\partial y_{i}}=-i \kappa \Lambda_{i} G_{\omega}
$$

where the subscript $i$ can be either 2 or 3 and corresponds to the tunnel transverse coordinates. Note that the no flow impedance boundary condition applies only to the case of no flow $(\mathrm{U}=0)$.

In sections 3.1 and 3.2 formulations for wind tunnels with rectangular and circular cross sections are derived. A schematic of these cross-sections is presented in Figure 3.

\subsection{Rectangular Cross-Section Wind Tunnel}

The following definitions are used in our formulation for a rectangular wind tunnel.

$$
\Psi_{n_{2}, n_{3}}=\psi_{n_{2}}\left(y_{2}\right) \psi_{n_{3}}\left(y_{3}\right) \quad \psi_{n_{i}}= \begin{cases}\cos \left(\frac{\pi k_{n_{i}}}{2 L_{i}}\left(y_{i}-d_{i}\right)\right) & \text { when } \quad n_{i}=0,2,4 \ldots \\ \sin \left(\frac{\pi k_{n_{i}}}{2 L_{i}}\left(y_{i}-d_{i}\right)\right) & \text { when } \quad n_{i}=1,3,5 \ldots\end{cases}
$$

and 


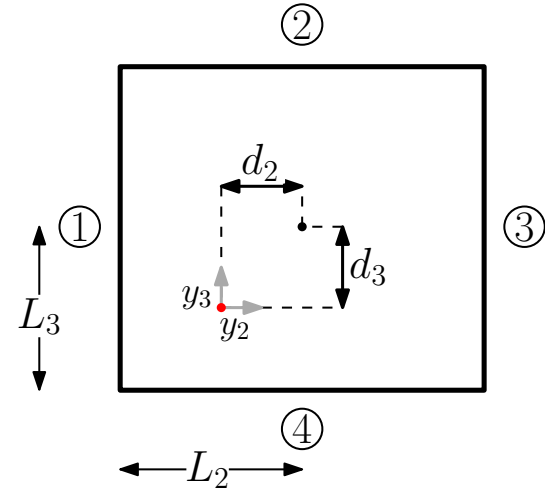

(a)

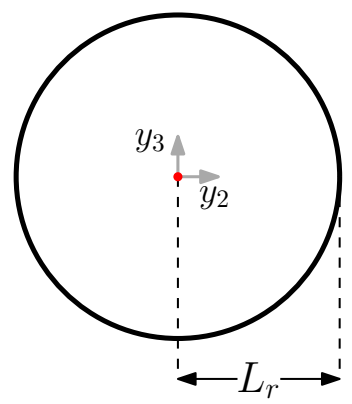

(b)

Figure 3: Schematic of (a) a rectangular and (b) a circular cross section wind tunnel. The parameters defining the shape and size of the tunnel cross-section are shown. For the rectangular cross-section schematic, the parameters describing the offset of the wind tunnel centreline from the origin of the co-ordinate system are also shown.

$$
\Gamma_{n_{2}, n_{3}}=\gamma_{n_{2}} \gamma_{n_{3}} \quad \gamma_{n_{i}}=\left\{\begin{array}{lll}
L_{i}\left[1+\operatorname{sinc}\left(k_{n_{i}}\right)\right] & \text { when } & n_{i}=0,2,4 \ldots \\
L_{i}\left[1-\operatorname{sinc}\left(k_{n_{i}}\right)\right] & \text { when } \quad n_{i}=1,3,5 \ldots
\end{array}\right.
$$

$L_{2}$ and $L_{3}$ are the half-width and half-height of the wind tunnel respectively whilst the parameters $d_{2}$ and $d_{3}$ are used to describe the offset of the origin from the wind tunnel centreline (see Figure 3 ) and

$$
-L_{i} \leq d_{i} \leq L_{i}
$$

From eq. (10) we can obtain a definition for the eigenvalue set in terms of the transverse wavenumbers $k_{n_{2}}$ and $k_{n_{3}}$

$$
\varkappa_{n_{2}, n_{3}}^{2}=\left(\frac{\pi k_{n_{2}}}{2 L_{2}}\right)^{2}+\left(\frac{\pi k_{n_{3}}}{2 L_{3}}\right)^{2}
$$

For rigid walls, the transverse wavenumbers are defined as

$$
k_{n_{i}}=n_{i} .
$$

To calculate the transverse wavenumbers for a wind tunnel with an impedance boundary, we solve the following expressions, derived from eq. (16) at each of the tunnel walls. For reference, a number has been assigned to each wall as presented in Figure 3. At walls 1 and 3

$$
\frac{\partial G_{\omega}}{\partial y_{2}}= \pm \mathrm{i} \kappa \Lambda_{2} G_{\omega} \quad \text { at } \quad y_{2}=d_{2} \mp L_{2}
$$

and at walls 2 and 4

$$
\frac{\partial G_{\omega}}{\partial y_{3}}=\mp \mathrm{i} \kappa \Lambda_{3} G_{\omega} \quad \text { at } \quad y_{3}=d_{3} \pm L_{3}
$$


In the following analysis we shall set $\Lambda_{2}=\Lambda_{3}=\Lambda$. Therefore, for a rectangular wind tunnel with impedance boundaries, the transverse wavenumbers can be determined using the following set of expressions

$$
\begin{array}{rcc}
\hat{k}_{i} \tan \hat{k}_{i}+\mathrm{i} \kappa \Lambda L_{i}=0 & \text { when } & n_{i}=0,2,4, \ldots \\
\hat{k}_{i} \cot \hat{k}_{i}-\mathrm{i} \kappa \Lambda L_{i}=0 & \text { when } & n_{i}=1,3,5, \ldots
\end{array}
$$

where $\hat{k}_{i}=\pi k_{n_{i}} / 2$. The transverse wavenumbers for a tunnel with an impedance boundary condition at its walls are complex and are not solved for analytically. The solutions are obtained by using a root finding technique and continuation from the rigid wall solution (where $\Lambda_{i}=0$ ) to the desired wall admittance. In the limit $\Lambda_{i} \rightarrow \infty$ the wavenumber is once again real-valued and would be equivalent to that required for a pressure release boundary. This iterative procedure, also known as a shooting method, has been discussed and used elsewhere ${ }^{7 ; 13}$.

\subsubsection{GRAF'S ADDITION THEOREM}

It will now be shown that the eigenfunctions specified in eq. (17) can be rewritten in cylindrical coordinates using Graf's addition theorem for Bessel functions ${ }^{14}$. This simplification provides a new expression for the Green's function which is convenient for evaluating the sound field produced by rotating sources. This new expression is then substituted into eq. (12) in order to determine the tonal noise produced by the blades of an open rotor in a rectangular wind tunnel with rigid walls.

Firstly, the eigenfunctions are expanded using the following relationships

$$
\begin{aligned}
& \cos \left(\frac{\pi k_{n_{i}}}{2 L_{i}}\left(y_{i}-d_{i}\right)\right)=\frac{1}{2}\left[\exp \left\{\mathrm{i} \frac{\pi k_{n_{i}}}{2 L_{i}} y_{i}-\mathrm{i} \frac{\pi k_{n_{i}}}{2 L_{i}} d_{i}\right\}+\exp \left\{-\mathrm{i} \frac{\pi k_{n_{i}}}{2 L_{i}} y_{i}+\mathrm{i} \frac{\pi k_{n_{i}}}{2 L_{i}} d_{i}\right\}\right], \\
& \sin \left(\frac{\pi k_{n_{i}}}{2 L_{i}}\left(y_{i}-d_{i}\right)\right)=\frac{1}{2 \mathrm{i}}\left[\exp \left\{\mathrm{i} \frac{\pi k_{n_{i}}}{2 L_{i}} y_{i}-\mathrm{i} \frac{\pi k_{n_{i}}}{2 L_{i}} d_{i}\right\}-\exp \left\{-\mathrm{i} \frac{\pi k_{n_{i}}}{2 L_{i}} y_{i}+\mathrm{i} \frac{\pi k_{n_{i}}}{2 L_{i}} d_{i}\right\}\right] .
\end{aligned}
$$

Then, converting to cylindrical polar co-ordinates and making use of the Jacobi-Anger expansions ${ }^{14}$ yields the following identities

$$
\begin{gathered}
\exp \left\{ \pm \mathrm{i} \frac{\pi k_{n_{2}}}{2 L_{2}} r \cos \phi\right\}=\sum_{q_{2}=-\infty}^{\infty} J_{q_{2}}\left(\frac{\pi k_{n_{2}} r}{2 L_{2}}\right) \exp \left\{\mathrm{i} q_{2}(\phi \pm \pi / 2)\right\} \\
\exp \left\{ \pm \mathrm{i} \frac{\pi k_{n_{3}}}{2 L_{3}} r \sin \phi\right\}=\sum_{q_{3}=-\infty}^{\infty} J_{q_{3}}\left(\frac{\pi k_{n_{3}} r}{2 L_{3}}\right) \exp \left\{ \pm \mathrm{i} q_{3} \phi\right\},
\end{gathered}
$$

where $J$ is a Bessel function of the first kind. Using eqs. (26)-(29), each of the terms in the double series of eigenfunctions can now be expressed as a series where each term is a combination of Bessel and exponential functions, taking the form of the following four expansions

$$
\begin{aligned}
\cos \left(\frac{\pi k_{n_{2}}}{2 L_{2}}\left(y_{2}-d_{2}\right)\right)=\frac{1}{2} \sum_{q_{2}=-\infty}^{\infty} J_{q_{2}}\left(\frac{\pi k_{n_{2}} r}{2 L_{2}}\right) & \exp \left\{\mathrm{i} q_{2} \phi\right\} \\
\times & {\left[\exp \left\{\mathrm{i} \frac{\pi q_{2}}{2}-\mathrm{i} \frac{\pi k_{n_{2}}}{2 L_{2}} d_{2}\right\}+\exp \left\{-\mathrm{i} \frac{\pi q_{2}}{2}+\mathrm{i} \frac{\pi k_{n_{2}}}{2 L_{2}} d_{2}\right\}\right], }
\end{aligned}
$$




$$
\begin{aligned}
& \sin \left(\frac{\pi k_{n_{2}}}{2 L_{2}}\left(y_{2}-d_{2}\right)\right)=\frac{1}{2 \mathrm{i}} \sum_{q_{2}=-\infty}^{\infty} J_{q_{2}}\left(\frac{\pi k_{n_{2}} r}{2 L_{2}}\right) \exp \left\{\mathrm{i} q_{2} \phi\right\} \\
& \times\left[\exp \left\{\mathrm{i} \frac{\pi q_{2}}{2}-\mathrm{i} \frac{\pi k_{n_{2}}}{2 L_{2}} d_{2}\right\}-\exp \left\{-\mathrm{i} \frac{\pi q_{2}}{2}+\mathrm{i} \frac{\pi k_{n_{2}}}{2 L_{2}} d_{2}\right\}\right], \\
& \cos \left(\frac{\pi k_{n_{3}}}{2 L_{3}}\left(y_{3}-d_{3}\right)\right)=\frac{1}{2} \sum_{q_{3}=-\infty}^{\infty} J_{q_{3}}\left(\frac{\pi k_{n_{3}} r}{2 L_{3}}\right) \exp \left\{\mathrm{i} q_{3} \phi\right\} \\
& \times\left[\exp \left\{-\mathrm{i} \frac{\pi k_{n_{3}}}{2 L_{3}} d_{3}\right\}+\exp \left\{\mathrm{i} \pi q_{3}+\mathrm{i} \frac{\pi k_{n_{3}}}{2 L_{3}} d_{3}\right\}\right], \\
& \sin \left(\frac{\pi k_{n_{3}}}{2 L_{3}}\left(y_{3}-d_{3}\right)\right)=\frac{1}{2 \mathrm{i}} \sum_{q_{3}=-\infty}^{\infty} J_{q_{3}}\left(\frac{\pi k_{n_{3}} r}{2 L_{3}}\right) \exp \left\{\mathrm{i} q_{3} \phi\right\} \\
& \times\left[\exp \left\{-\mathrm{i} \frac{\pi k_{n_{3}}}{2 L_{3}} d_{3}\right\}-\exp \left\{\mathrm{i} \pi q_{3}+\mathrm{i} \frac{\pi k_{n_{3}}}{2 L_{3}} d_{3}\right\}\right] .
\end{aligned}
$$

Therefore

$$
\psi_{n_{2}}=\frac{1}{2} \sum_{q_{2}=-\infty}^{\infty} J_{q_{2}}\left(\frac{\pi k_{n_{2}} r}{2 L_{2}}\right) \exp \left\{\mathrm{i} q_{2} \phi\right\} Q_{n_{2}, q_{2}}
$$

where

$$
Q_{n_{2}, q_{2}}=\left\{\begin{array}{l}
{\left[\exp \left\{\mathrm{i} \frac{\pi q_{2}}{2}-\mathrm{i} \frac{\pi k_{n_{2}}}{2 L_{2}} d_{2}\right\}+\exp \left\{-\mathrm{i} \frac{\pi q_{2}}{2}+\mathrm{i} \frac{\pi k_{n_{2}}}{2 L_{2}} d_{2}\right\}\right]} \\
-\mathrm{i}\left[\exp \left\{\mathrm{i} \frac{\pi q_{2}}{2}-\mathrm{i} \frac{\pi k_{n_{2}}}{2 L_{2}} d_{2}\right\}-\exp \left\{-\mathrm{i} \frac{\pi q_{2}}{2}+\mathrm{i} \frac{\pi k_{n_{2}}}{2 L_{2}} d_{2}\right\}\right] \quad \text { when } \quad n_{2}=0,2,4 \ldots \\
n_{2}=1,3,5 \ldots
\end{array}\right.
$$

and

$$
\psi_{n_{3}}=\frac{1}{2} \sum_{q_{3}=-\infty}^{\infty} J_{q_{3}}\left(\frac{\pi k_{n_{3}} r}{2 L_{3}}\right) \exp \left\{\mathrm{i} q_{3} \phi\right\} \hat{Q}_{n_{3}, q_{3}}
$$

where

$$
\hat{Q}_{n_{3}, q_{3}}=\left\{\begin{array}{l}
{\left[\exp \left\{-\mathrm{i} \frac{\pi k_{n_{3}}}{2 L_{3}} d_{3}\right\}+\exp \left\{\mathrm{i} \pi q_{3}+\mathrm{i} \frac{\pi k_{n_{3}}}{2 L_{3}} d_{3}\right\}\right] \quad \text { when } \quad n_{3}=0,2,4 \ldots} \\
-\mathrm{i}\left[\exp \left\{-\mathrm{i} \frac{\pi k_{n_{3}}}{2 L_{3}} d_{3}\right\}-\exp \left\{\mathrm{i} \pi q_{3}+\mathrm{i} \frac{\pi k_{n_{3}}}{2 L_{3}} d_{3}\right\}\right] \quad \text { when } \quad n_{3}=1,3,5 \ldots
\end{array}\right.
$$

Combining these expressions yields a new expression for the source eigenfunctions but in cylindrical coordinates:

$$
\Psi_{n_{2}, n_{3}}=\frac{1}{4} \sum_{q_{2}=-\infty}^{\infty} \sum_{q_{3}=-\infty}^{\infty} J_{q_{2}}\left(\frac{\pi k_{n_{2}} r}{2 L_{2}}\right) J_{q_{3}}\left(\frac{\pi k_{n_{3}} r}{2 L_{3}}\right) \exp \left\{\mathrm{i}\left(q_{2}+q_{3}\right) \phi\right\} Q_{n_{2}, q_{2}} \hat{Q}_{n_{3}, q_{3}} .
$$


We shift to using the summation index $q=q_{3}+q_{2}$. Making use of the identity $J_{-\nu}(z)=J_{\nu}(z) \exp \{\mathrm{i} \nu \pi\}$ and the symmetry of the infinite summation in $q_{2}$ then yields the following altered expressions

$$
\Psi_{n_{2}, n_{3}}=\frac{1}{4} \sum_{q_{2}=-\infty}^{\infty} \sum_{q=-\infty}^{\infty} J_{q_{2}}\left(\frac{\pi k_{n_{2}} r}{2 L_{2}}\right) J_{q_{2}+q}\left(\frac{\pi k_{n_{3}} r}{2 L_{3}}\right) \exp \left\{\mathrm{i} q \phi+\mathrm{i} q_{2} \pi\right\} Q_{n_{2},-q_{2}} \hat{Q}_{n_{3}, q+q_{2}}
$$

Note that Graf's Addition Theorem ${ }^{14}$ states

$$
\sum_{q_{2}=-\infty}^{\infty} J_{q_{2}}\left(\frac{\pi k_{n_{2}} r}{2 L_{2}}\right) J_{q+q_{2}}\left(\frac{\pi k_{n_{3}} r}{2 L_{3}}\right) \exp \left\{ \pm \mathrm{i} q_{2} \pi / 2\right\}=J_{q}\left(k_{r} r\right) \exp \{ \pm \mathrm{i} q \chi\}
$$

where

$$
\begin{gathered}
k_{r}=\sqrt{\left(\frac{\pi k_{n_{2}}}{2 L_{2}}\right)^{2}+\left(\frac{\pi k_{n_{3}}}{2 L_{3}}\right)^{2}}=\varkappa_{n_{2}, n_{3}}, \\
\tan \chi=\frac{k_{n_{2}}}{k_{n_{3}}} \frac{L_{3}}{L_{2}} .
\end{gathered}
$$

Combining all the terms that are dependent on the summation index $q_{2}$ and using Graf's addition theorem results in an expression for the eigenfunction that is only dependent on one summation index, $q$. For simplicity the remaining phase terms are combined into a single term, $\mathcal{Q}_{n_{2}, n_{3}, q}\left(\chi, d_{2}, d_{3}\right)$, where the form of $\mathcal{Q}$ will be dependent on whether $n_{i}$ is odd or even. The expression for the eigenfunctions can now be redefined in terms of cylindrical source co-ordinates with only one summation,

$$
\Psi_{n_{2}, n_{3}}=\frac{1}{4} \sum_{q=-\infty}^{\infty} J_{q}\left(k_{r} r\right) \exp \{\mathrm{i} q \phi\} \mathcal{Q}_{n_{2}, n_{3}, q}
$$

Substituting eq. (43) into eq. (12) yields the following new (and convenient) expression for the frequency domain Green's function of an infinitely long rectangular cross section wind tunnel which is suited to modelling rotor sources:

$$
\begin{aligned}
G_{\omega}(\mathbf{y} \mid \mathbf{x})=\frac{-\mathrm{i}}{8} \sum_{n_{2}=0}^{\infty} \sum_{n_{3}=0}^{\infty} \sum_{q=-\infty}^{\infty} \frac{J_{q}\left(k_{r} r\right) \mathcal{Q}_{n_{2}, n_{3}, q} \Psi_{n_{2}, n_{3}}\left(x_{2}, x_{3}\right)}{\Gamma_{n_{2}, n_{3}} k_{n_{2}, n_{3}}} \\
\exp \left\{\mathrm{i} q \phi-\mathrm{i} \frac{M \kappa}{\beta^{2}}\left(y_{1}-x_{1}\right)-\mathrm{i} \frac{k_{n_{2}, n_{3}}}{\beta^{2}}\left|y_{1}-x_{1}\right|\right\} .
\end{aligned}
$$

This Green's function is equivalent to the original expression where the source co-ordinates were instead expressed using a rectangular co-ordinate system (obtained by substituting eq. (17) into eq. (12)).

\subsubsection{Expressions for Rotor Loading Sources}

We first introduce the following Fourier transform pair for the loading term $L^{(b)}$

$$
L^{(b)}\left(X^{(b)}, r, \tau\right)=\int_{-\infty}^{\infty} \tilde{L}^{(b)}\left(X^{(b)}, r, \omega^{\prime}\right) \exp \left\{\mathrm{i} \omega^{\prime} \tau\right\} \mathrm{d} \omega^{\prime}
$$




$$
\tilde{L}^{(b)}\left(X^{(b)}, r, \omega^{\prime}\right)=\frac{1}{2 \pi} \int_{-\infty}^{\infty} L^{(b)}\left(X^{(b)}, r, \tau\right) \exp \left\{-\mathrm{i} \omega^{\prime} \tau\right\} \mathrm{d} \tau
$$

Then, combining the expressions given in eqs. (6), (7), (8), (11), (44) and (45) yields eq. (47) (after further simplification) for the acoustic pressure in the wind tunnel.

$$
\begin{aligned}
& p(\mathbf{x}, t)= \frac{-1}{8} \sum_{n_{2}=0}^{\infty} \sum_{n_{3}=0}^{\infty} \sum_{q=-\infty}^{\infty} \sum_{b=0}^{B-1} \int_{R_{h}}^{R_{t}} \int_{-c / 2}^{c / 2} \int_{-\infty}^{\infty} \tilde{L}^{(b)}\left(X^{(b)}, r, \omega-q \Omega\right) \\
& {\left[\sin \alpha\left(\frac{M \kappa \pm k_{n_{2}, n_{3}}}{\beta^{2}}\right)-q \frac{\cos \alpha}{r}\right] \frac{J_{q}\left(k_{r} r\right) \mathcal{Q}_{n_{2}, n_{3}, q} \Psi_{n_{2}, n_{3}}\left(x_{2}, x_{3}\right)}{\Gamma_{n_{2}, n_{3}} k_{n_{2}, n_{3}}} } \\
& \exp \left\{\mathrm{i} \omega t+\mathrm{i} q \frac{2 \pi b}{B}+\mathrm{i}\left(\frac{M \kappa \pm k_{n_{2}, n_{3}}}{\beta^{2}}\right) x_{1}-\mathrm{i}\left[\left(\frac{M \kappa \pm k_{n_{2}, n_{3}}}{\beta^{2}}\right) \cos \alpha+q \frac{\sin \alpha}{r}\right]\left(s+X^{(b)}\right)\right\} \\
& \mathrm{d} \omega \mathrm{d} X^{(b)} \mathrm{d} r .
\end{aligned}
$$

This is a general equation which can be used to predict the acoustic pressure due to the lift forces on the blades of an open rotor installed in a rectangular cross-section wind tunnel and observed at a location $\mathbf{x}$ either upstream (upper sign) or downstream (lower sign) of the source.

The frequency domain pressure jump due to $B$ evenly spaced rotating point forces of magnitude $F$ Newtons, located at radius $a$ and aligned normal to the local flow direction can be expressed as

$$
\tilde{L}^{(b)}\left(X^{m}, r, \omega\right)=F \delta(r-a) \delta\left(X^{(b)}\right) \delta(\omega) .
$$

Substituting eq. (48) into eq. (47) and evaluating the summation over $b$ yields the following expression

$$
\begin{aligned}
p(\mathbf{x}, t)=-\frac{B F}{8} \sum_{n_{2}=0}^{\infty} \sum_{n_{3}=0}^{\infty} \sum_{q=-\infty}^{\infty} & {\left[\sin \alpha\left(\frac{M \kappa \pm k_{n_{2}, n_{3}}}{\beta^{2}}\right)-\nu \frac{\cos \alpha}{a}\right] } \\
& \frac{J_{\nu}\left(k_{r} a\right) \mathcal{Q}_{n_{2}, n_{3}, \nu} \Psi_{n_{2}, n_{3}}\left(x_{2}, x_{3}\right)}{\Gamma_{n_{2}, n_{3}} k_{n_{2}, n_{3}}} \exp \left\{\mathrm{i} \nu \Omega t+\mathrm{i}\left(\frac{M \kappa \pm k_{n_{2}, n_{3}}}{\beta^{2}}\right) x_{1}\right\} .
\end{aligned}
$$

where $\nu=q B, \kappa=\nu \Omega / c_{0}$ and we have arbitrarily set $s=0$. Each value of $q$ corresponds to a tone harmonic generated by the rotor source.

The general expression for rotor loading sources given in eq. (47) may also be used to calculate the noise generated by distributed sources which more accurately simulate a rotor. Eq. (47) is used here to calculate the noise generated by steady loading on the blades of an open rotor and by the unsteady loading due to interactions between the wakes emanating from an upstream contra-rotating rotor and the blades on a downstream rotor.

For steady loading, the Fourier transform of the loading term is given by

$$
\tilde{L}^{(b)}\left(X^{m}, r, \omega\right)=\frac{1}{2} \rho_{0} U_{r}^{2} C_{L} f\left(X^{(b)}\right) \delta(\omega) .
$$

where $U_{r}$ is the speed of the rotor blade relative to the fluid, $C_{L}$ is the local lift coefficient of the blade section and here $f$ is a dimensionless function which describes the chordwise distribution of lift. Substituting eq. (50) into eq. (47) and evaluating the summation over $b$ then yields the following expression for the acoustic pressure 
generated by steady loading on the blades of an Open Rotor situated in a wind tunnel with a rectangular cross-section:

$$
\begin{aligned}
p(\mathbf{x}, t)=\frac{-B \rho_{0}}{16} \sum_{n_{2}=0}^{\infty} \sum_{n_{3}=0}^{\infty} \sum_{q=-\infty}^{\infty} \frac{\mathcal{Q}_{n_{2}, n_{3}, \nu} \Psi_{n_{2}, n_{3}}\left(x_{2}, x_{3}\right)}{\Gamma_{n_{2}, n_{3}} k_{n_{2}, n_{3}}} & \\
& \times \exp \left\{\mathrm{i} \nu \Omega t+\mathrm{i}\left(\frac{M \kappa \pm k_{n_{2}, n_{3}}}{\beta^{2}}\right) x_{1}\right\} \int_{R_{h}}^{R_{t}} J_{\nu}\left(k_{r} r\right) U_{r}^{2} c C_{L} \Psi_{L} k_{Y} \exp \left\{-\mathrm{i} \phi_{s}\right\} \mathrm{d} r .
\end{aligned}
$$

Here $c$ is the rotor blade chord and is a function of blade radius, $\nu=q B, \kappa=\nu \Omega / c_{0}, \bar{X}^{(b)}=X^{(b)} / c$,

$$
\begin{aligned}
& \phi_{s}=\left[\left(\frac{M \kappa \pm k_{n_{2}, n_{3}}}{\beta^{2}}\right) \cos \alpha+\nu \frac{\sin \alpha}{r}\right] s, \\
& k_{c}=\left[\left(\frac{M \kappa \pm k_{n_{2}, n_{3}}}{\beta^{2}}\right) \cos \alpha+\nu \frac{\sin \alpha}{r}\right] c, \\
& k_{Y}=\left[\left(\frac{M \kappa \pm k_{n_{2}, n_{3}}}{\beta^{2}}\right) \sin \alpha-\nu \frac{\cos \alpha}{r}\right],
\end{aligned}
$$

and

$$
\Psi_{L}=\int_{-1 / 2}^{1 / 2} f\left(X^{(b)}\right) \exp \left\{-\mathrm{i} k_{c} \bar{X}^{(b)}\right\} \mathrm{d} \bar{X}^{(b)}
$$

A model for noise generated by the unsteady loading on the blades of the rear rotor, which is caused by contra-rotating wakes generated at the front rotor, is now presented. The unsteady loading caused by the periodic distortion and on a single blade, here indexed by $b=0$, is first expressed as a Fourier Series

$$
L^{(0)}\left(X^{(0)}, r, \tau\right)=\sum_{k=-\infty}^{\infty} L_{k}^{(0)}\left(X^{(0)}, r\right) \exp \left\{\mathrm{i} k B_{1}\left(\Omega_{1}+\Omega_{2}\right) \tau\right\}
$$

where

$$
L_{k}^{(0)}\left(X^{(0)}, r\right)=\frac{1}{T} \int_{0}^{T} L^{(0)}\left(X^{(0)}, r, \tau\right) \exp \left\{-\mathrm{i} k B_{1}\left(\Omega_{1}+\Omega_{2}\right) \tau\right\} \mathrm{d} \tau
$$

and the period $T=2 \pi /\left(B_{1}\left(\Omega_{1}+\Omega_{2}\right)\right)$.

Note that the loading on the $b^{\text {th }}$ blade is related to the reference blade by

$$
\begin{aligned}
& L^{(b)}\left(X^{(b)}, r, \tau\right)=L^{(0)}\left(X^{(b)}, r, \tau+\frac{2 \pi b}{B_{2}\left(\Omega_{1}+\Omega_{2}\right)}\right) \\
& \quad=\sum_{k=-\infty}^{\infty} L_{k}^{(0)}\left(X^{(b)}, r\right) \exp \left\{\mathrm{i} k B_{1}\left(\Omega_{1}+\Omega_{2}\right) \tau+2 \pi \mathrm{i} b k \frac{B_{1}}{B_{2}}\right\}
\end{aligned}
$$


and so

$$
\begin{aligned}
\tilde{L}^{(b)}\left(X^{(b)}, r, \omega\right)=\frac{1}{2 \pi} \int_{-\infty}^{\infty} \sum_{k=-\infty}^{\infty} L_{k}^{(0)} & \left(X^{(b)}, r\right) \exp \left\{-\mathrm{i}\left(\omega-k B_{1}\left(\Omega_{1}+\Omega_{2}\right)\right) \tau+2 \pi \mathrm{i} b k \frac{B_{1}}{B_{2}}\right\} \mathrm{d} \tau \\
& =\sum_{k=-\infty}^{\infty} L_{k}^{(0)}\left(X^{(b)}, r\right) \exp \left\{2 \pi \mathrm{i} b k \frac{B_{1}}{B_{2}}\right\} \delta\left(\omega-k B_{1}\left(\Omega_{1}+\Omega_{2}\right)\right)
\end{aligned}
$$

Substituting eq. (59) into eq. (47) and evaluating the summation over $b$ results in the following expression for the acoustic pressure.

$$
\begin{aligned}
p(\mathbf{x}, t)=\frac{-B_{2}}{8} \sum_{n_{2}=0}^{\infty} \sum_{n_{3}=0}^{\infty} \sum_{q=-\infty}^{\infty} \sum_{k=-\infty}^{\infty} \frac{\mathcal{Q}_{n_{2}, n_{3}, \nu} \Psi_{n_{2}, n_{3}}\left(x_{2}, x_{3}\right)}{\Gamma_{n_{2}, n_{3}} k_{n_{2}, n_{3}}} \\
\quad \exp \left\{\mathrm{i}\left(q B_{2} \Omega_{2}+k B_{1} \Omega_{1}\right) t+\mathrm{i}\left(\frac{M \kappa \pm k_{n_{2}, n_{3}}}{\beta^{2}}\right) x_{1}\right\} \int_{R_{h}}^{R_{t}} J_{\nu}\left(k_{r} r\right) c \Psi_{L} k_{Y} \exp \left\{-\mathrm{i} \phi_{s}\right\} \mathrm{d} r .
\end{aligned}
$$

For this expression, $\nu=q B_{2}-k B_{1}, \kappa=\left(q B_{2} \Omega_{2}+k B_{1} \Omega_{1}\right) / c_{0}$,

$$
\Psi_{L}=\int_{-1 / 2}^{1 / 2} L_{k}^{(0)}\left(X^{(b)}, r\right) \exp \left\{-\mathrm{i} k_{c} \bar{X}^{(b)}\right\} \mathrm{d} \bar{X}^{(b)}
$$

and the other parameters are defined as before, taking into account the fact that, in this case, sound is radiated from the rear propeller.

The benefit of using Graf's addition theorem arises from the potential improvements in speed. Using a rectangular co-ordinate system for the Green's function results in an expression that contains an extra $\phi$ integral which would need to be evaluated numerically. By converting the expression to cylindrical coordinates, via Graf's addition theorem, the $\phi$ integral is instead evaluated analytically. The benefits obtained in either speed or accuracy will thus arise from the margin of error to which the numerical integral is evaluated and ultimately is determined by the number of function calls that are required by the integration routine being used. Assuming an acceptable margin of error, the amount of speed up will also depend on a number of other modelling parameters as well, such as for example the observer position or evaluated mode count. As an example, a comparable calculation was conducted using both forms of the expression. An adaptive GaussKronod quadrature routine implemented in MATLAB was used for numerical integration. When generating a pressure contour at constant axial position near the plane of the rotor, such as those presented in this paper, a calculation speed up of three orders of magnitude was noted when using the new formulation.

\subsection{Circular Cross-Section Wind Tunnel}

Similar expressions may also be easily derived for a wind tunnel with a circular cross-section. A diagram representing this cross-section was also presented in Figure 3; in this case the radius to the tunnel wall is denoted by $L_{r}$. In this case the rotor axis and wind tunnel axis are collinear. The frequency domain Green's function is still of similar form to that presented in eq. (12) but it is more convenient to formulate the expression in cylindrical co-ordinates, such that 


$$
G_{\omega}(\mathbf{y} \mid \mathbf{x})=-\frac{\mathrm{i}}{2} \sum_{m} \sum_{n} \frac{\Psi_{m n}^{*}\left(\phi_{x}, r_{x}\right) \Psi_{m n}\left(\phi_{y}, r_{y}\right)}{\Gamma_{m n} k_{m n}} \exp \left\{-\mathrm{i}\left[\frac{M \kappa}{\beta^{2}}\left(y_{1}-x_{1}\right)+\frac{k_{m n}}{\beta^{2}}\left|x_{1}-y_{1}\right|\right]\right\}
$$

where the summation indices $n_{2}$ and $n_{3}$ have been exchanged for $m$ and $n$ to differentiate them from the previous case and here we have allowed for the possibility of complex eigenfunctions. The eigenfunction $\Psi_{m n}$ is again a solution to eq. (10) over the cross section of the duct and expressing this in cylindrical co-ordinates leads to

$$
\left(\frac{1}{r_{y}^{2}} \frac{\partial^{2}}{\partial \phi_{y}^{2}}+\frac{1}{r_{y}} \frac{\partial}{\partial r_{y}}+\frac{\partial^{2}}{\partial r_{y}^{2}}+\varkappa_{m n}^{2}\right) \Psi_{m n}=0
$$

As previously, a wall boundary condition must also be satisfied, which in this case is only dependant on the radial co-ordinate $r$. For example, for a rigid wall wind tunnel, the boundary condition is

$$
\frac{\partial \Psi_{m n}}{\partial r}=0
$$

For a cylindrical wind tunnel, we use the following definition for the eigenfunction set

$$
\Psi_{m n}(r, \phi)=J_{m}\left(\varkappa_{m n} r\right) \exp \{\mathrm{i} m \phi\}
$$

As before $\varkappa_{m n}$ can be determined by using the boundary condition, so for the rigid wall case these are quite easily determined by finding the zeros of the derivative of the Bessel function $J$. Note that for each order $m$ of the Bessel function, there will be an infinite number of zeros to find, which are specified by the index $n$. The zeros are found by using an iterative search routine (for example using a bi-section method), in a similar fashion to the method required for finding the eigenvalues of a rectangular wind tunnel with an impedance boundary. Also in this case, when the orthogonality condition is satisfied,

$$
\Gamma_{m n}=\int_{A} \Psi_{m n} \Psi_{m n}^{*} \mathrm{~d} A=\int_{0}^{L_{r}} \int_{0}^{2 \pi} J_{m}\left(\varkappa_{m n} r_{y}\right) J_{m}^{*}\left(\varkappa_{m n} r_{y}\right) \exp \left\{\mathrm{i}(m-m) \phi_{y}\right\} r_{y} \mathrm{~d} \phi_{y} \mathrm{~d} r_{y}
$$

and again making use of the rigid wall boundary condition to solve the integral over $r$ (and the fact that in our analysis we only consider real values of $\varkappa_{m n}$ ) then yields

$$
\begin{aligned}
& \Gamma_{m, n}=\frac{1}{4}\left[\phi_{y}+\frac{\sin \left(2 m \phi_{y}\right)}{2 m}\right]_{0}^{2 \pi}\left[\frac{r_{y}^{2} \varkappa_{m, n}^{2}-m^{2}}{\varkappa_{m, n}^{2}} J_{n}^{2}\left(\varkappa_{m, n} r_{y}\right)\right]_{0}^{L_{r}} \\
& =\frac{\pi\left(L_{r}^{2} \varkappa_{m, n}^{2}-m^{2}\right)}{2 \varkappa_{m, n}^{2}}(1+\operatorname{sinc}(4 \pi m)) J_{n}^{2}\left(\varkappa_{m, n} L_{r}\right) \\
& \Gamma_{m n}=2 \pi \int_{0}^{L_{r}} J_{m}^{2}\left(\varkappa_{m n} r_{y}\right) r_{y} \mathrm{~d} r_{y}=\left[\frac{r_{y}^{2}}{2}\left[J_{m}^{\prime}\left(\varkappa_{m n} r_{y}\right)\right]^{2}+\frac{r_{y}^{2}}{2}\left(1-\frac{m^{2}}{\left(\varkappa_{m n} r_{y}\right)^{2}}\right)\left[J_{m}\left(\varkappa_{m n} r_{y}\right)\right]^{2}\right]_{0}^{L_{r}} \\
& =\frac{L_{r}^{2}}{2}\left(1-\frac{m^{2}}{\left(\varkappa_{m n} L_{r}\right)^{2}}\right)\left[J_{m}\left(\varkappa_{m n} L_{r}\right)\right]^{2}=\frac{L_{r}^{2} \varkappa_{m n}^{2}-m^{2}}{2 \varkappa_{m n}^{2}}\left[J_{m}\left(\varkappa_{m n} L_{r}\right)\right]^{2}
\end{aligned}
$$

Substituting these terms into eq. (62) gives 


$$
G_{\omega}(\mathbf{y} \mid \mathbf{x})=-\frac{\mathrm{i}}{2} \sum_{m=-\infty}^{\infty} \sum_{n=0}^{\infty} \frac{J_{m}\left(\varkappa_{m n} r_{y}\right) J_{m}\left(\varkappa_{m n} r_{x}\right)}{k_{m n} \Gamma_{m n}} \exp \left\{-\mathrm{i}\left[K^{ \pm}\left(x_{1}-y_{1}\right)-m\left(\phi_{y}-\phi_{x}\right)\right]\right\}
$$

It is possible to generate a similar expression to represent a wind tunnel with an impedance wall, by instead using an impedance boundary condition. Green's functions for such cylindrical geometries are covered extensively in the literature, for example in Rienstra and Tester ${ }^{15}$.

\subsubsection{Expression for Rotor Loading Sources}

Substituting eqs. (6), (7), (11), (69) and (45) into eq. (8) yields the following general expression for the acoustic pressure produced by loading sources representing an open rotor in a circular wind tunnel.

$$
\begin{aligned}
p(\mathbf{x}, t)= & \frac{1}{2} \sum_{b=1}^{B} \sum_{m=-\infty}^{\infty} \sum_{n=0}^{\infty} \int_{r_{h}}^{r_{t}} \int_{-c / 2}^{c / 2} \int_{-\infty}^{\infty}\left[K_{1}^{ \pm} \sin \alpha+\frac{m \cos \alpha}{r}\right] \frac{\tilde{L}\left(X^{(b)}, r, \omega-m \Omega\right) J_{m}\left(\varkappa_{m n} r_{y}\right) J_{m}\left(\varkappa_{m n} r_{x}\right)}{k_{m n} \Gamma_{m n}} \\
& \times \exp \left\{\mathrm{i} \omega t-\mathrm{i} K^{ \pm} x_{1}-\mathrm{i} m \phi_{x}+\frac{2 \pi \mathrm{i} b m}{B}+\mathrm{i}\left[K_{1}^{ \pm} \cos \alpha+\frac{m \sin \alpha}{r}\right]\left(s+X^{(b)}\right)\right\} \mathrm{d} \omega \mathrm{d} X \mathrm{~d} r_{y}
\end{aligned}
$$

For a rotating point force, set $s=0$ (zero sweep) and substitute eq. (48) for $\tilde{L}$. Solving the remaining integrals and the summation over $b$ gives

$$
p(\mathbf{x}, t)=\sum_{m=-\infty}^{\infty} p_{m} \exp \{\mathrm{i} \nu \Omega t\}
$$

where

$$
p_{m}=\frac{B F}{2} \sum_{n=0}^{\infty}\left[K_{1}^{ \pm} \sin \alpha+\frac{\nu \cos \alpha}{a}\right] \frac{J_{\nu}\left(\varkappa_{m n} a\right) J_{\nu}\left(\varkappa_{m n} r_{x}\right)}{\Gamma_{m n} k_{m n}}, \exp \left\{-\mathrm{i} \nu \phi_{x}-\mathrm{i} K_{1}^{ \pm} x_{1}\right\}
$$

$\nu=m B$ and $\kappa=\nu \Omega / c_{0}$. An expression for the tonal noise generated by the steady loading rotor source can be derived by substituting eq. (50) into eq. (70), which after some manipulation yields

$$
\begin{aligned}
p_{m}=\frac{B \rho_{0}}{4} \sum_{n=-\infty}^{\infty} \frac{J_{\nu}\left(\varkappa_{m n} r_{x}\right)}{\Gamma_{m n} k_{m n}} \exp \{ & \left.-\mathrm{i}\left(\frac{-M \kappa \pm k_{m n}}{\beta^{2}}\right) x_{1}-\mathrm{i} \nu \phi_{x}\right\} \\
& \times \int_{R_{h}}^{R_{t}}\left[K_{1}^{ \pm} \sin \alpha+\frac{\nu \cos \alpha}{r_{y}}\right] J_{\nu}\left(\varkappa_{m n} r_{y}\right) c U_{r}^{2} C_{L} \Psi_{L} \exp \left\{-\mathrm{i} \phi_{s}\right\} \mathrm{d} r_{y}
\end{aligned}
$$

where $\nu$ and $\kappa$ are defined as before, but $k_{c}$ is now defined as

$$
k_{c}=\left[K_{1}^{ \pm} \cos \alpha+\frac{\nu \sin \alpha}{r}\right] c
$$

with $\phi_{s}=k_{c} s / c$. An expression for the acoustic pressure caused by unsteady loading on the rear rotor, due to its interaction with the wakes from the front rotor, can be derived in a similar fashion using eq. (59) and yielding 


$$
\begin{aligned}
& p(\mathbf{x}, t)=\frac{B_{2}}{2} \sum_{m=-\infty}^{\infty} \sum_{k=-\infty}^{\infty} \sum_{n=0}^{\infty} \frac{J_{\nu}\left(\varkappa_{m n} r_{x}\right)}{\Gamma_{m n} k_{m n}} \exp \left\{\mathrm{i} \omega_{k m} t-\mathrm{i}\left(\frac{-M \kappa \pm k_{\nu, n}}{\beta^{2}}\right) x_{1}-\mathrm{i} \nu \phi_{x}\right\} \\
& \times\left[K_{1}^{ \pm} \sin \alpha+\frac{\nu \cos \alpha}{r}\right] \int_{R_{h}}^{R_{t}} J_{\nu}\left(\varkappa_{m n} r_{y}\right) c \Psi_{L} \exp \left\{\mathrm{i} \phi_{s}\right\} \mathrm{d} r_{y}
\end{aligned}
$$

where now $\nu=m B_{2}-k B_{1}, \omega=k B_{1} \Omega_{1}+m B_{2} \Omega_{2}$ and $\Psi_{L}$ is defined by eq. (61).

\section{Analysis}

Eqs. (49) and (71) are used to understand the impact of wind tunnel reverberation on the noise generated by rotating point forces and assess what effect the impedance of the wind tunnel walls and the shape of the wind tunnel cross section may have on the reverberant noise field. This point force model is a simplification of the problem of rotor tone noise in a wind tunnel environment. However it has been shown that in the free-field such a model produces a reasonably accurate representation of a propeller noise field ${ }^{16}$.

The effect of tunnel wall impedance on the acoustic field generated by a set of 12 rotating point forces is demonstrated in Figure 4, which plots sound pressure level (SPL) versus axial distance from the plane of rotation at a sideline radius of $0.75 \mathrm{~m}$. The radius of rotation is $0.35 \mathrm{~m}$ and the rotation Mach number, $M_{s}$, of the point forces is 0.5 , where $M_{s}=a \Omega / c_{0}$. This generates a fundamental tone with a frequency of $950 \mathrm{~Hz}$. The rectangular cross section wind tunnel model is used with a purely resistive impedance set equal to the characteristic specific acoustic impedance of air (i.e. $\Lambda=1$ ). The tunnel cross section has side lengths of $2.02 \mathrm{~m}$ and $2.33 \mathrm{~m}$; this produces a cross-section equivalent to that in the test section of the afore-mentioned ARA Transonic Wind Tunnel. Note that, in this case, there is no flow in the wind tunnel.

In Figure 4 the finite impedance tunnel model is compared to a free-field model (i.e. with no scattering objects present). The free-field pressure of a single frequency component is given by

$$
p_{m}(\mathbf{x}, t)=\frac{B F}{8 \pi} \int_{-\infty}^{\infty}\left[k_{1} \sin \alpha-\frac{\nu \cos \alpha}{a}\right] J_{\nu}\left(k_{r} r_{<}\right) H_{\nu}^{(2)}\left(k_{r} r_{>}\right) \exp \left\{\mathrm{i} \nu \Omega t+\mathrm{i} \nu \phi_{x}-\mathrm{i} k_{1} x_{1}\right\} \mathrm{d} k_{1}
$$

where $\nu=m B$ (and $m$ corresponds to the tone harmonic index), the radial wavenumber $k_{r}$ is defined by the dispersion relation

$$
k_{r}=\sqrt{\left(\kappa-k_{1} M\right)^{2}-k_{1}^{2}}
$$

and $r_{<}$is the smaller of $r_{x}$ and $r_{y}$ whilst $r_{>}$corresponds to the larger of the two. All the other parameters are consistent with previous definitions.

Figure 4 shows how, for both axially oriented rotating point forces and circumferentially oriented rotating point forces in a wind tunnel, the acoustic field close to the source plane is in reasonable agreement with that observed in the free-field. This is an expected result, since for incidence angles roughly normal to the wall the acoustic impedance is almost equivalent to the characteristic specific acoustic impedance of air. Therefore, for this case, measurements taken in a region close to the rotor disc plane would be free from contamination by the reverberant noise field.

Under ideal conditions, the acoustic liner insert designed for Open Rotor testing in the ARA wind tunnel would achieve similar results to the example case demonstrated in Figure 4. However, at high flow speeds $(M>0.5)$, it is believed that performance of the acoustic liner is degraded, particularly upstream of the 


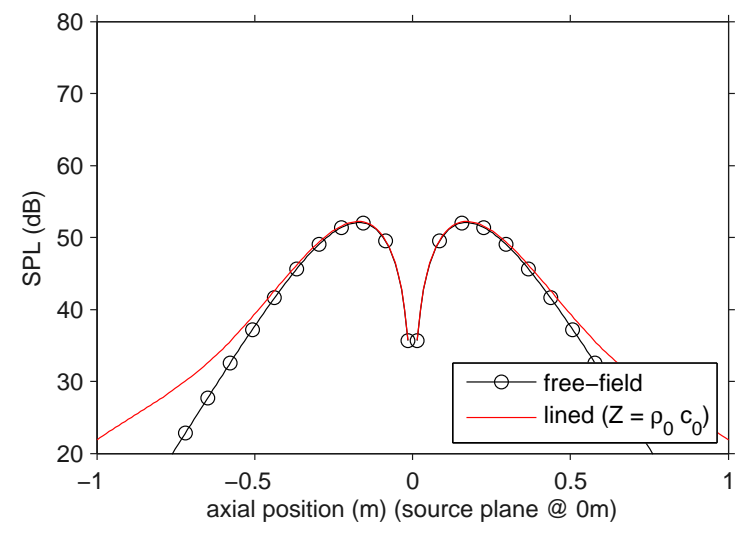

(a)

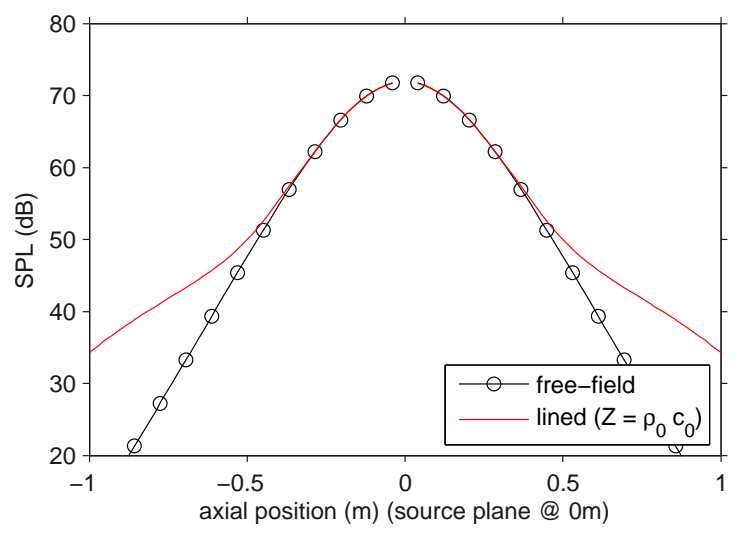

(b)

Figure 4: Directivities produced by (a) axial point forces and (b) tangential point forces. Here, the specific acoustic impedance of the lined wind tunnel model (red) is set to 1 and compared with a free-field point force model (black).

source. Degradation in the liner's performance at high flow speeds has also been noted in previous research ${ }^{1}$ and thus, as an initial approximation, the walls are modelled as rigid instead. McAlpine describes the validity of a similar approximation at high speed (and frequency) for the application of acoustic liners in mitigating buzz-saw tone noise ${ }^{17}$.

Figure 5 (a) shows a comparison of predicted sound pressure levels against radius for rigid wall wind tunnel models using both square and circular cross-sections and the acoustic pressure is produced by a rotor modelled by 12 rotating point forces located at the propeller tip and aligned perpendicular to the local flow direction relative to the blade at that location. The forces rotate at a radius of $1 \mathrm{~m}$ and the rotation speed of the point forces is $1000 \mathrm{RPM}$, equivalent to a source rotational Mach number of 0.3 . There is no axial flow in this case. The Helmholtz number for the fundamental tone generated by the point forces in this circular wind tunnel is 37. All Helmholtz numbers provided in this paper are based on a circular wind tunnel casing radius, $L_{r}$, which in this case is $10 \mathrm{~m}$. These predictions are for sound levels at locations close to the plane of rotation $\left(x_{1}=0.01 \mathrm{~m}, y_{1}=0 \mathrm{~m}\right)$. An equivalent free-field prediction is also included. A schematic showing the relative size and location of the rotating point forces within the wind tunnels is shown in Figure 5 (b). In this case the diameter of the cross-section of the circular wind tunnel is $20 \mathrm{~m}$ and is equal to the side length of the square cross-section wind tunnel. Note that accurate calculation of the noise field using the wind tunnel models on the source plane requires evaluation of the infinite modal sum (which is computationally expensive due to the large number of cut-off modes which contribute to the noise field at these locations). However, as the axial distance of the observation position from the plane of rotation increases, the number of cut-off modes which need to be included in the summation drastically reduces.

The acoustic field presented is typical of a subsonic rotating source - there is a region close to the source in which the sound pressure decays exponentially. In this region the direct field of the sources is dominant for the wind tunnel solutions since they are in agreement with the free-field solution. Outboard of this region however, the impact of the tunnels' reverberant noise fields is noticeable and a reverberation pattern forms. The bounding radius of this region is consistent with the definition of the Mach radius ${ }^{16}$,

$$
r_{m}=\frac{a \sqrt{1-M^{2} \sin ^{2} \theta}}{M_{s} \sin \theta}
$$

which, in the plane of the rotor, simplifies to the definition of the sonic radius ${ }^{18}$, 


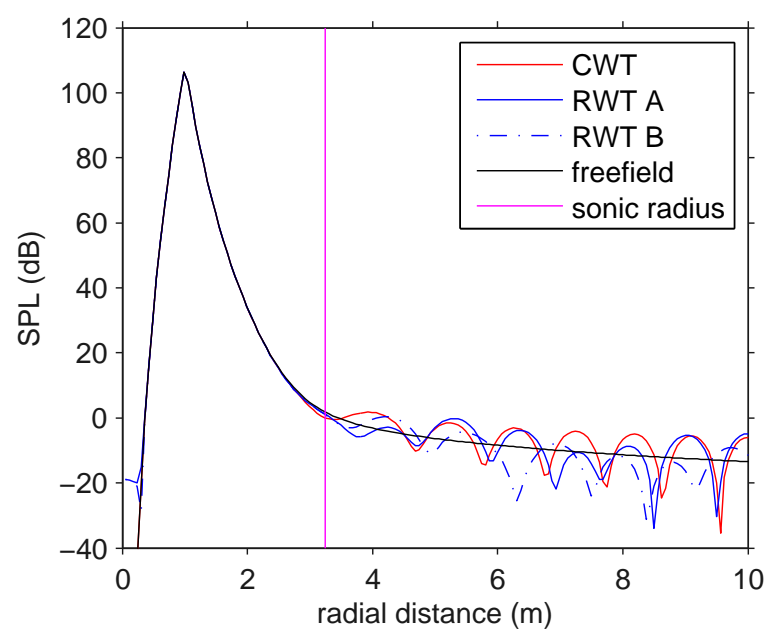

(a)

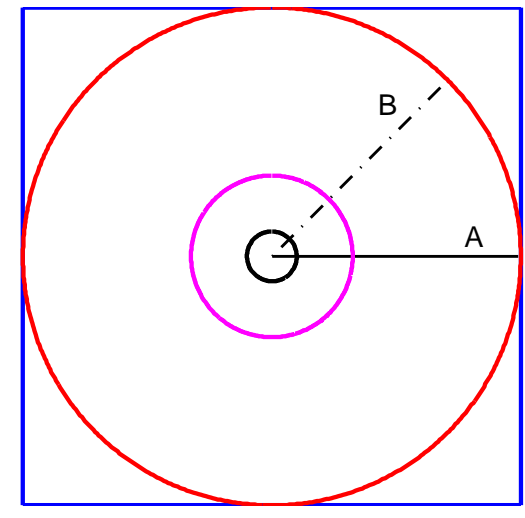

(b)

Figure 5: Comparison of the rectangular (RWT) and circular (CWT) cross section wind tunnel models with a free-field model for the sound generated by rotating point dipoles, shown on (a). The dimensions for both tunnels are represented in (b) along with the source radius (black) and sonic radius of the source (magenta). The arrays on which the RWT model were evaluated are represented by the letters A and B. The fields generated by the CWT and free-field models are axisymmetric

$$
r_{s}=\frac{a \sqrt{1-M^{2}}}{M_{s}}
$$

where again, $M$ is the (axial) flow Mach number and the variable $M_{s}$ is the rotational Mach number of the source. The polar observer angle, $\theta$, has also been introduced.

For no axial flow $(M=0)$, the sonic radius is $a / M_{s}$ ( $a$ being the radius of the rotating forces). The sonic radius is a useful concept in rotor acoustics since in the plane of the rotor it separates regions in which the free-field acoustic radiation is evanescent (inboard) and 'sustained' (outboard). Note how the field exhibits 'far-field' behaviour outboard of the sonic radius, where decay of the pressure amplitude is inversely proportional to the sideline distance. However inboard of the sonic radius the field exhibits 'near-field' behaviour and decay from the source radius occurs at a faster rate. Most importantly, the amplitude of acoustic pressure of the direct noise field is much larger than that of the reverberant field in the 'near-field' region, which is why a reverberation pattern fails to form in this region and the tunnel solutions are well matched to the free-field. The essentially uncontaminated field in the rotor's near-field may be used to accurately quantify the noise output of the rotor. This information may also be extrapolated to predict rotor noise at far-field positions. An extrapolation tool is described in Peake and Boyd ${ }^{16}$ and such a tool has already been applied to measurements from this test rig, further documented in Parry et $\mathrm{al}^{3}$.

An obvious difference between both wind tunnel models is in the regularity in the reverberation pattern of the level and position of the lobes generated. The circular wind tunnel solution has a fairly regular pattern of lobes and since the field is axisymmetric this does not change with the azimuthal angle of the radial array. Similar observations are not true for the rectangular cross-section model. The pattern is dependant on azimuthal angle (as seen by differences in the results generated at arrays A and B in Figure 5) and furthermore the regularity in the features of the lobes that is demonstrated in the circular wind tunnel solution is not present in the rectangular one, even for array A, at which the tunnel wall is normal and at the same distance from the source as in the circular model. Array A and B are selected arbitrarily in this case and simply to demonstrate the variation with azimuthal angle for the field in the square cross-section wind tunnel. The reverberation pattern across the whole cross-section of a rectangular wind tunnel can be 
quite complex and is presented and discussed in the next section of this paper.

\subsection{Simulation of the ARA's Transonic Wind Tunnel}

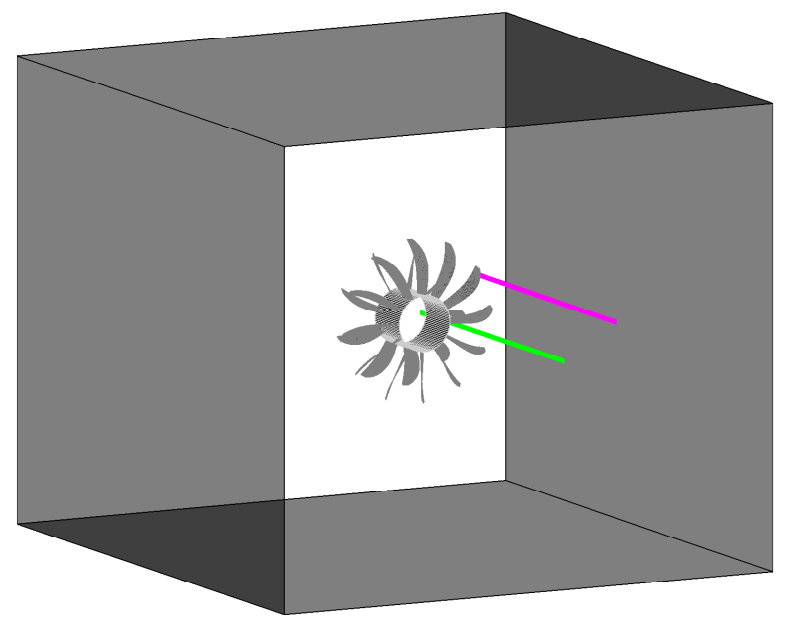

Figure 6: Diagram representing Rolls-Royce's Rig 145 in the ARA wind tunnel in Bedford. Measurement rails at typical puller and pusher sidelines have also been included.

Figure 6 is a simplified schematic of Rig 145 in ARA's Transonic Wind Tunnel test section (with the acoustic liner inserted). Also included in the figure are two measurement rails, which are placed at sideline distances equivalent to the location of the outer surface of the fuselage of a passenger aircraft for Open Rotor installations in both the pusher and puller configuration (after accounting for model scaling). Obtaining measurements at these sideline distances would be useful for predicting the level of noise inside the passenger cabin during cruise flight on an Open Rotor powered aircraft.

The contours in Figure 7 (a) and (b) respectively show the sound pressure level of the fundamental tone produced by 10 rotating forces, aligned normal to the local flow direction, in a rectangular cross section wind tunnel with rigid walls and walls with an impedance equal to $\rho_{0} c_{0}$. The radius of rotation is $0.35 \mathrm{~m}$ and the rotation speed Mach number is 0.61 . This generates a fundamental tone frequency at $955 \mathrm{~Hz}$. These parameters are chosen to approximately simulate the sound pressure level of a $[1,0]$ tone (i.e. the blade passing frequency tone of the first rotor) that would be generated by a model scale rotor placed in ARA's Transonic Wind Tunnel (as shown in Figure 6). The result for a rigid wall wind tunnel in Figure 7 (a) gives a visible indication of the complex reverberation pattern that is generated for rotating sources in a rectangular wind tunnel. A rotational symmetry of order 2 is noted on the cross-section, but other than this there is no obvious pattern to the reverberant field, which makes prediction of the impact of reverberation on the rotor noise field difficult. In contrast, imposing an impedance $Z=\rho_{0} c_{0}$ on the walls, as in the contour shown in Figure 7 (b), significantly reduces the effect of reverberation. The difference in the SPL generated by these two models is presented in Figure 7 (c). The contour qualitatively shows that differences in the two acoustic fields are clearer closer to the wind tunnel walls. The fields become more similar towards the source radius, where the SPL delta tends to zero. The acoustic field generated by the 'lined' walls in Figure 7 closely (but not precisely) resembles the field generated by equivalent rotating sources in the free-field. The difference in SPL generated by the 'lined' wind tunnel model and an equivalent free-field model is shown in Figure 7 (d). The acoustic field inboard of the source radius has been masked in Figure 7 (c) and (d). The differences due 
to wind tunnel reverberation in this region are larger and are also not of interest since it is not practical to take measurements in this region. This region is therefore masked to improve visualisation of the acoustic field outboard of the source radius. 


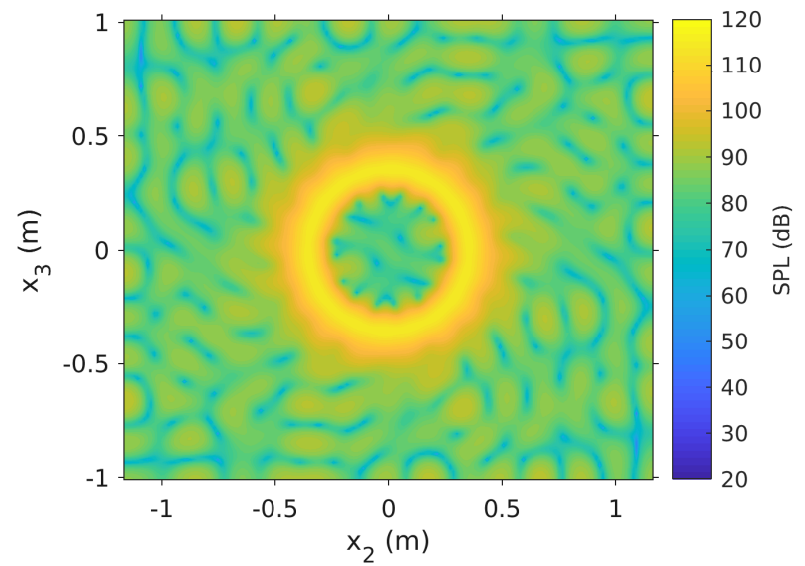

(a)

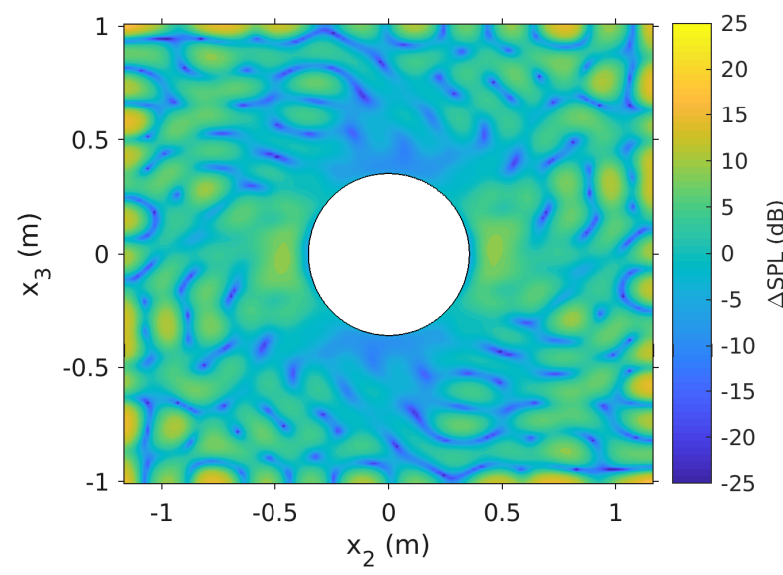

(c)

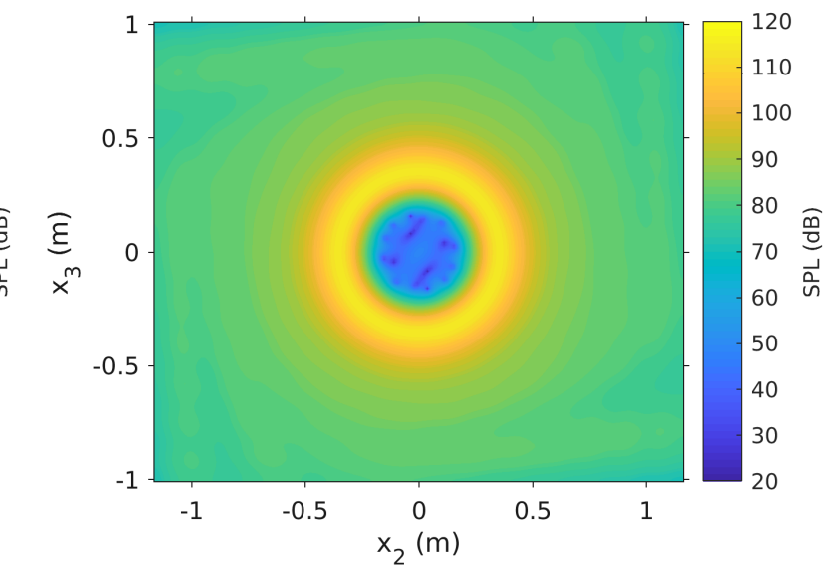

(b)

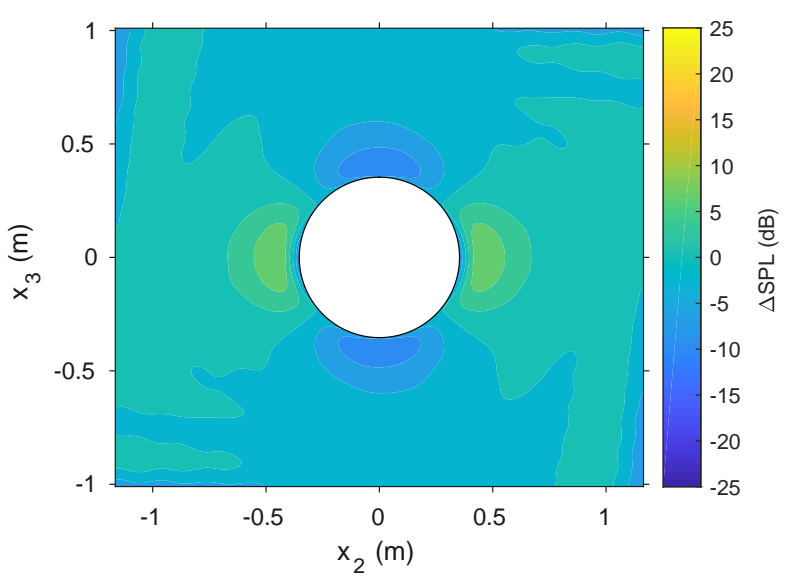

(d)

Figure 7: Contours of the sound pressure level of the fundamental tone generated by 10 rotating point forces, over the cross section of ARA's transonic wind tunnel with (a) rigid walls and (b) walls with impedance $Z=\rho_{0} c_{0}$ (i.e. purely resistive). The plane of the contour $\left(x_{1}=0.01 \mathrm{~m}\right)$ is close to the source plane $\left(y_{1}=0 \mathrm{~m}\right)$. The difference in SPL between the two predicted fields is presented in (c). The difference in SPL between the solution with 'lined' walls and an equivalent free-field prediction is presented in (d). 


\subsection{Simulations of ONERA's S1MA wind tunnel}

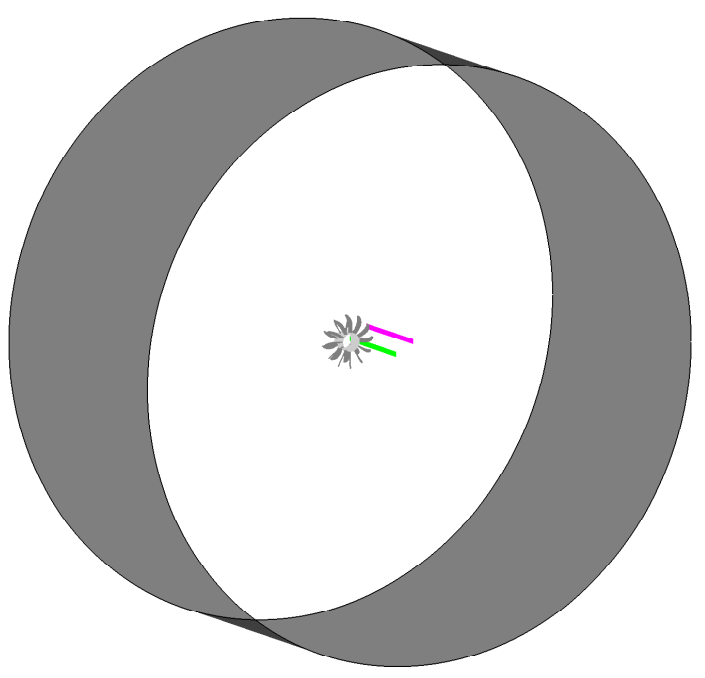

Figure 8: Diagram representing Rolls-Royce's Rig 145 in the S1MA wind tunnel in Modane. Measurement rails at typical puller and pusher sidelines have also been included.

Figure 8 is a schematic approximating the installation of a model scale open rotor test rig in ONERA's S1MA wind tunnel test section, which has a diameter of $8 \mathrm{~m}$. In this analysis the tunnel walls are assumed to be perfectly rigid and the test rig is installed exactly at the tunnel centreline. Note that to date, testing of Rolls-Royce's open rotor test rig, Rig 145, has never been conducted in this wind tunnel. However the S1MA wind tunnel is also a possible choice for conducting high speed Open Rotor noise experiments, since it is capable of flow speeds up to Mach 1. The aim of this analysis is to derive simple estimates for the potential effect of reverberation on Open Rotor tone noise. Comparison of this schematic with Figure 6 indicates how much larger the cross section is in this wind tunnel. Again, two measurement rails corresponding to passenger cabin sideline distances for pusher and puller Open Rotor installation are included.

The sound pressure level contour in Figure 9 shows how in a perfectly cylindrical wind tunnel with rigid walls the field amplitude is axisymmetric. The results in this figure are generated by a set of 10 rotating point forces aligned normal to the local flow direction, this time at a rotation radius of $0.5 \mathrm{~m}$, rotating at a Mach number of 0.875 and again generating a fundamental tone with a frequency of $955 \mathrm{~Hz}$ (leading to a Helmholtz number of approximately 70). The reverberant pattern in the wind tunnel is much simpler in structure than that observed in a rectangular cross section wind tunnel (Figure 7). However it is still apparent that for this case at some radial distance outboard of the source, reverberation contaminates the field.

The circular wind tunnel results are also compared with a free-field solution in Figure 10, where results are instead shown along a radial path in the same plane $\left(x_{1}=0.01 \mathrm{~m}\right)$. The figure also includes the location of the measurement rails as well as the location of the sonic radius (eq. (79)).

At these operating conditions, the rotor speed is at the lower end of its typical operating range. This coupled with zero flow speed mean that the sonic radius is placed towards its outer limit for the operating conditions of this test rig. The wind tunnel solution does not match the free-field solution outboard of the sonic radius, however the levels are an almost perfect match inboard. The far-field levels of the two solutions are approximately similar, however it is quite clear that reverberation from the rigid tunnel walls has had an impact on the noise field in this outer region. The deviations in the wind tunnel prediction oscillate around the free-field solution, the range of which seems to expand towards the tunnel wall. This is believed to be 


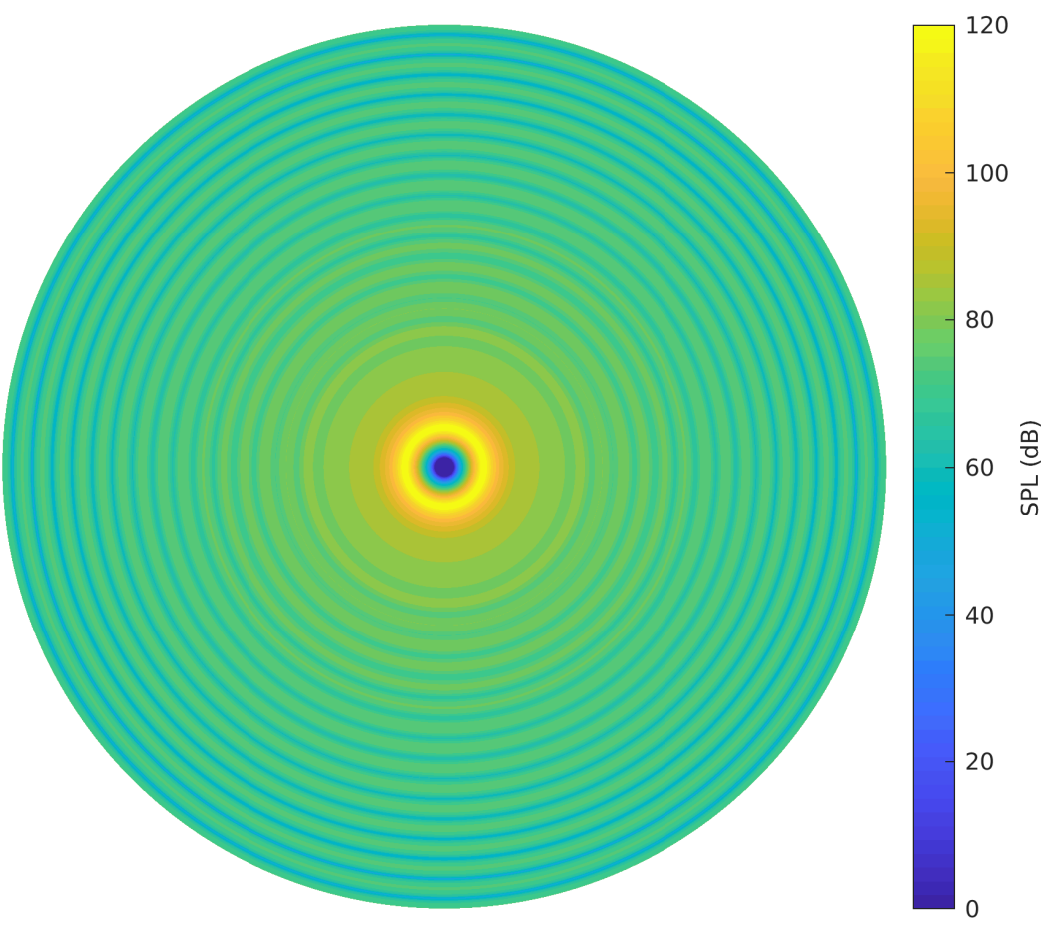

Figure 9: Contour of the sound pressure level generated by 10 rotating point forces, on the cross section of the S1MA wind tunnel. The contour plane $\left(x_{1}=0.01 \mathrm{~m}\right)$ is close to the source plane $\left(y_{1}=0 \mathrm{~m}\right)$, in order to assess near-field noise radiation in the tunnel.

due to interference between the direct and reflected sound fields, the levels of which are most comparable at the tunnel wall.

The conclusion then is that the sonic radius is a key factor in determining the extent of the reverberant field in a circular cross section wind tunnel. If the measurement rails are placed inboard of the sonic radius it may be expected that the result obtained in a wind tunnel may be consistent with that expected in the free-field.

In Figure 11, it is shown that the reverberation pattern exists in the entire volume of the wind tunnel except in the near-field region, where the radiation is evanescent. Figure 11 shows a sound pressure level contour for the same case as before, however the contour plane has been rotated to span the tunnel radius and axis. Here the contours now represent the sound pressure level delta between the wind tunnel and free-field solutions. The contour focuses on the source region. The thick black line represents the radial extent of the source, whereas the region in white corresponds to where the wind tunnel solution was not evaluated due to computational expense. The red line indicates the boundary of the near-field region $r_{n}$ defined by

$$
r_{n}=\sqrt{r_{m}^{2}-\frac{r_{m}^{2} x_{1}^{2}}{r_{m}^{2}-a^{2}}} .
$$

This relation for the boundary was derived by analysis of the complex phase function in Prentice's rotor noise formulation and is discussed further in his paper ${ }^{19}$. It is shown that inboard of this boundary the radiation is evanescent whilst outboard radiation is sinusoidal. Again, the conclusion that can be drawn from this result is that the direct noise field of the source has a significantly larger amplitude in the rotor's 'near-field' 


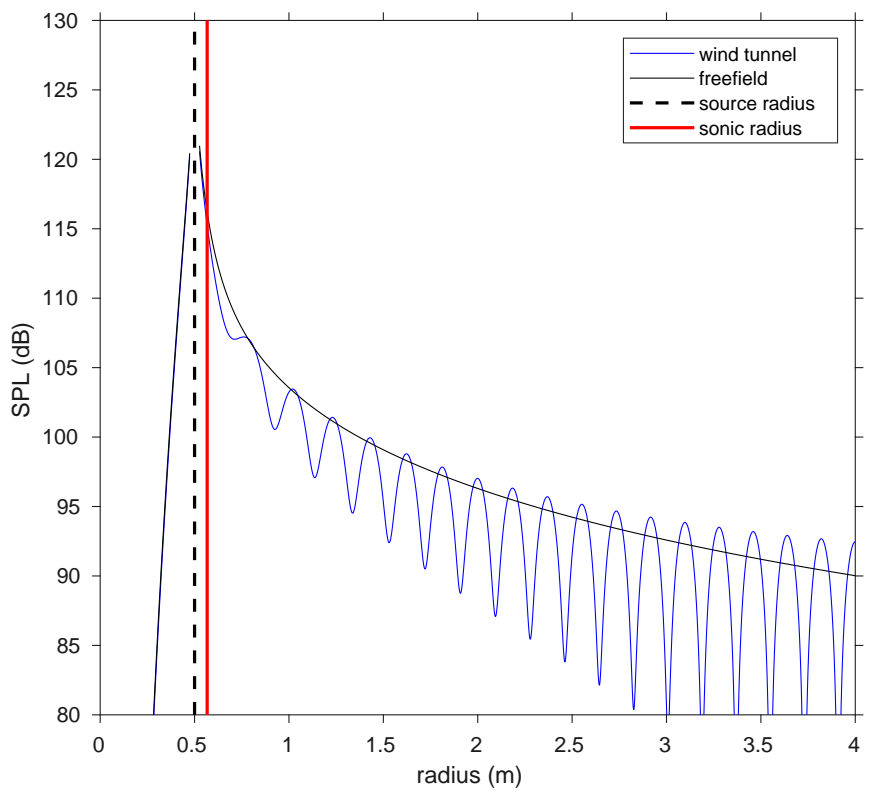

Figure 10: Simulations of a $[1,0]$ tone generated by a typical model scale open rotor rig setup, in the freefield and in the S1MA wind tunnel, with no flow. The results presented are radial directivities of the sound pressure level.

region and so dominates the reverberant noise field. In the plane of the rotor source, the near-field boundary $r_{n}$ is equivalent to the sonic radius $r_{s}$.

Next, the effect of flow is considered in Figure 12. The same parameters used to generate the results shown in Figure 7 are again applied in this case except that now the flow Mach number $M=0.7$, which is a typical cruise flight Mach number. The additional flow speed increases the relative Mach number of the source and thus reduces the sonic radius (see eq. (79)). As shown in Figure 12, the sonic radius is now inboard of both rail 1 and rail 2 and at these 'measurement' positions the impact of tunnel reverberation has resulted in the wind tunnel solution deviating from the free-field solution. This result suggests that for a typical cruise condition test point for an open rotor test rig, such as Rig 145, and in this wind tunnel setup, measurements made at these sideline distances should not be assumed to be equivalent to noise levels in the free-field.

Note an increase in rotor speed would draw the sonic radius further inboard. The source radii considered in this paper are considered representative of a model scale rotor tip radius. In such a case, it would not be possible to obtain measurements inboard of the source radius and near the source plane of the rotor due to the rotor's physical presence. It is possible then that during such Open Rotor test campaigns, obtaining measurements in the rotor near-field could actually be impossible.

Figure 13 plots the sound pressure level versus radial location for an observer close to the plane of rotation for the first tone produced by 4 evenly spaced point forces located at radius $0.4 \mathrm{~m}$ and rotating at a Mach number of 2.9. The tone has an azimuthal mode order of 4 and a frequency of $1590 \mathrm{~Hz}$. Results are presented for free-field radiation and radiation inside the S1MA wind tunnel. Such a tone is equivalent to the first interaction tone produced by a contra-rotating open rotor with 12 blades on the front rotor and 8 on the rear rotor, with both rotors having a tip Mach number of 0.58 .

Interaction tones typically have a much higher mode phase speed than the rotor-alone tones produced by a counter-rotation Open Rotor. The effective source rotation Mach number ${ }^{16}, M_{s}^{\prime}$, of the equivalent set of rotating point forces is defined as 


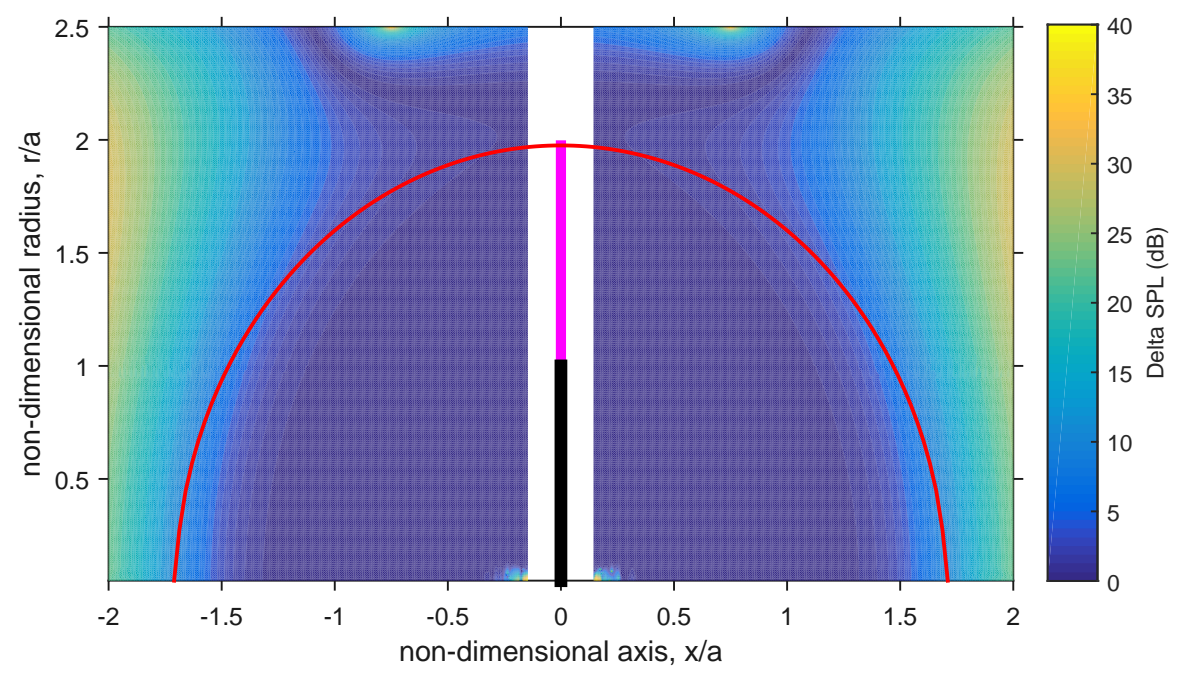

Figure 11: An axially and radially varying contour showing the delta in sound pressure level between wind tunnel and free-field solutions. The results simulate a $[1,0]$ tone generated by a typical open rotor rig setup and the S1MA wind tunnel. All distances have been made non-dimensional using the source radius, $a$. The red line indicates the near-field boundary of the rotor source, as predicted by eq (80).

$$
M_{s}^{\prime}=\frac{a\left(k B_{k} \Omega_{k}+m B_{m} \Omega_{m}\right)}{c_{0}\left(k B_{k}-m B_{m}\right)}
$$

and the effective azimuthal mode number, $\nu^{\prime}$, (i.e. the number of rotating point forces required for the tone of interest) is given by

$$
\nu^{\prime}=k B_{k}-m B_{m}
$$

where $k$ and $m$ respectively correspond to harmonics of the front and rear rotor and the subscript $k$ and $m$ to parameters of the front and rear rotor. The interaction tone sonic radius is shown in Figure 13. It is apparent from the figure that the reverberant noise field distorts the direct noise field right up to the source radius and so at any viable measurement position, interaction tones will always be affected by tunnel reverberation. For typical Open Rotor operating conditions, the sonic radius of an interaction tone will usually occur inboard of the tip radius and so it is likely that interaction tone measurements in a rigid wall wind tunnel will not generally by representative of free-field measurements. To demonstrate this, the figure also shows suggested measurement rail positions, which lie outboard of the sonic radius and thus in the region impacted by reverberation. $\mathrm{s}$ 


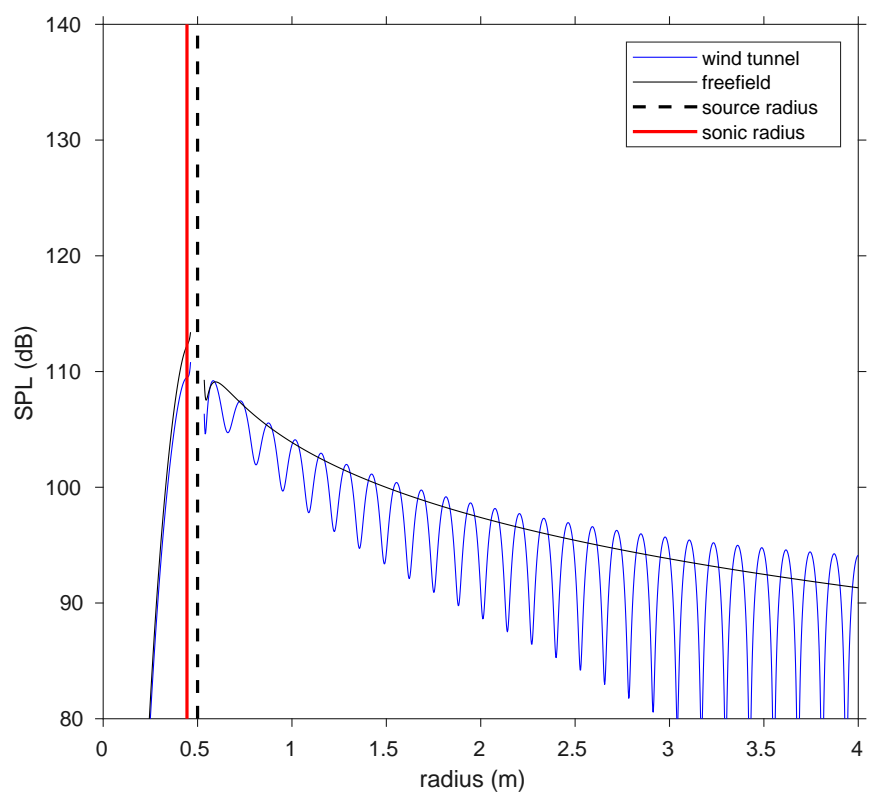

Figure 12: Simulations of the [1,0] tone generated by a typical open rotor rig setup, in the free-field and in the S1MA wind tunnel, with flow Mach number $M=0.7$. The results presented are radial directivities of the sound pressure level.
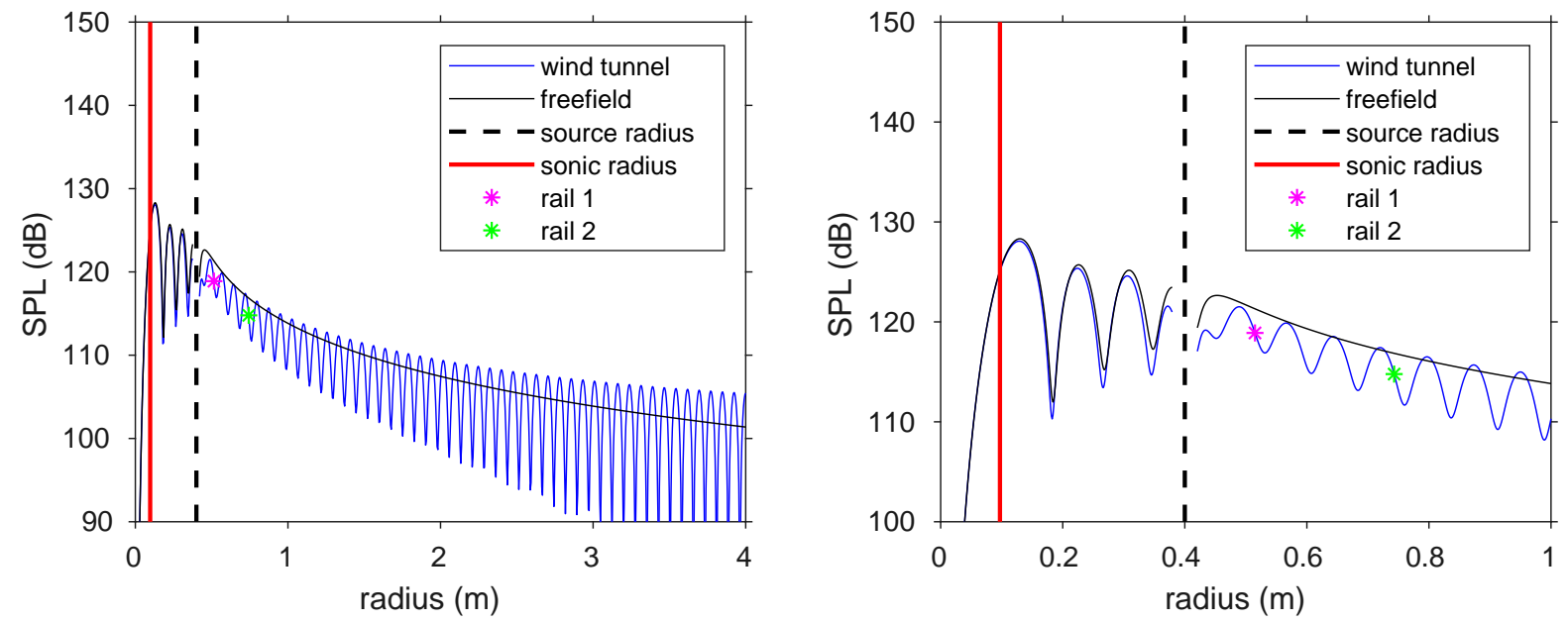

Figure 13: Simulations of the $[1,1]$ interaction tone generated by Rig 145 in the free-field and in the S1MA wind tunnel, with flow Mach number $M=0.7$. The results presented are radial directivities of the sound pressure level, where (a) show the directivity over the entire tunnel radius whilst (b) focuses on the source region. 


\section{Conclusions}

Simple analytical models for the tone noise generated by Open Rotors, situated in either circular or rectangular cross-section wind tunnels, have been presented. Graf's addition theorem was used to simplify the expression for the acoustic field within the rectangular wind tunnel, and was shown to greatly reduce the computation cost of the model. The models were relatively simple, and were for tunnels with rigid walls and a constant mean-flow or were for tunnels with no-flow and walls with finite impedance.

The analysis presented in this paper indicates that the sound pressure generated by an Open Rotor in a wind tunnel with rigid walls may not be representative of that generated in the free-field, unless measured in the acoustic near-field of the rotor where the direct field dominates the waves reflected from the wind tunnel walls. However, for many tones generated by an open rotor at cruise conditions, it may be difficult or even impossible to make measurements in this region.

The models presented in this paper could be extended to investigate wind tunnel reverberation in further detail. A model that accounts for both impedance and a mean flow simultaneously is a natural extension and would remove the modelling assumption that the tunnel walls behave rigidly at high flow speeds. Modifying the plug flow assumption to allow variation in the mean flow across the wind tunnel cross section (especially close to the wind tunnel walls) may provide a more accurate prediction, however this is dependent on frequency. A combination of both these modelling improvements would allow for the study of possible shielding on the acoustic liner, an effect which would certainly have an impact on noise measurements. The improved understanding from such work would translate to more accurate predictions of cruise regime noise of Open Rotor powered aircraft.

A further extension of this model would be to include other rotor noise sources. This paper has focussed on open rotor tone noise however rotor broadband noise sources are also of interest, yet it has proven challenging to validate prediction against measurements. Furthermore, broadband noise measurements in high flow speeds would also require the use of closed section wind tunnels and so the radiated noise will be susceptible to reverberation. A number of rotor broadband noise prediction models exist in the literature and these often consider the radiation to be formed by rotating dipoles. Sources defined in this manner could be combined with the Green's functions presented in this paper to form the required analytic expression. The difficulty in broadband noise prediction would arise from computing other modelling parameters, such as the blade surface pressure fluctuation (particularly at high speed) and boundary layer turbulence convection velocity ${ }^{20}$. Also, the expense of such a computation would be strongly dependent on both the number of frequencies of interest and the number of modes required for calculation convergence at each frequency.

\section{Acknowledgements}

The authors would like to acknowledge Rolls-Royce plc. and the Engineering and Physical Sciences Research Council (EPSRC) for funding the EngD project under which this work was carried out. The primary author would also like to thank Dr A McAlpine, Dr P Murray and Dr G Gabard for helpful discussions regarding this work. The authors also recognise that much of this work was only made possible because of industrial collaborations between Rolls-Royce plc, the Aircraft Research Association and the Institute of Sound and Vibration Research.

\section{References}

[1] T. J. Kirker. Procurement and testing of a $1 / 5$ scale advanced counter rotating propfan model. In 13th AIAA Aeroacoustics Conference, 1990.

[2] M. E. Wood and D. A. Newman. The design and commissioning of an acoustic liner for propeller noise testing in the ARA transonic wind tunnel. IMechE, 1990. 
[3] A. B. Parry, K. Britchford, M. J Kingan, and P. Sureshkumar. Aeroacoustic Tests of Isolated Open Rotors at High Speed. In 18th AIAA Aeroacoustics Conference, 2012.

[4] D. B. Hanson. Noise of counter-rotation propellers. Journal of Aircraft, 22:609-617, July 1985. ISSN 0021-8669. URL http://adsabs .harvard.edu/abs/1985JAir . .22 . .609H.

[5] Donald B. Hanson. Nearfield Frequency-Domain Theory for Propeller Noise. AIAA Journal, 23(4), 1985.

[6] Donald B. Hanson. Direct frequency domain calculation of open rotor noise. AIAA Journal, 30(9):2334-2337, September 1992. ISSN 0001-1452. doi: 10.2514/3.11223. URL http://doi.aiaa.org/10.2514/3.11223.

[7] Sjoerd W. Rienstra and Brian J. Tester. An analytic Green's function for a lined circular duct containing uniform mean flow. Journal of Sound and Vibration, 317(3-5): 994-1016, November 2008. ISSN 0022460X. doi: 10.1016/j.jsv.2008.03.048. URL http://linkinghub.elsevier.com/retrieve/pii/S0022460X08002836.

[8] Jose S. Alonso and Ricardo a. Burdisso. Green's Functions for the Acoustic Field in Lined Ducts with Uniform Flow. AIAA Journal, 45(11):2677-2687, November 2007. ISSN 0001-1452. doi: 10.2514/1.29872. URL http://arc.aiaa.org/doi/abs/10.2514/1.29872.

[9] E.J. Brambley and N. Peake. Classification of aeroacoustically relevant surface modes in cylindrical lined ducts. Wave Motion, 43(4):301-310, April 2006. ISSN 01652125. doi: 10.1016/j.wavemoti.2006.01.001. URL http://linkinghub.elsevier.com/retrieve/pii/S0165212506000023.

[10] Edmane Envia. Aeroacoustic Analysis of a High-Speed Open Rotor. International Journal of Aeroacoustics, 14(3-4):569-606, aug 2015. ISSN 1475-472X. doi: 10.1260/1475-472X.14.3-4.569. URL http://journals. sagepub.com/doi/10.1260/1475-472X.14.3-4.569.

[11] Michael J Kingan and Prathiban Sureshkumar. Open Rotor Centrebody Scattering. Journal of Sound and Vibration, 333:418-433, 2013.

[12] M Goldstein. Aeroacoustics. 1976.

[13] A. McAlpine and M. C. M. Wright. Acoustic scattering by a spliced turbofan inlet duct liner at supersonic fan speeds. Journal of Sound and Vibration, 292(35):911-934, May 2006. ISSN 0022460X. doi: 10.1016/j.jsv.2005.09.010. URL http://linkinghub.elsevier.com/retrieve/pii/S0022460X05006577.

[14] M. Abramowitz and I. A. Stegun. Handbook of Mathematical Functions. National Bureau of Standards, 1972.

[15] Sjoerd Rienstra and Brian Tester. An Analytic Green's Function for a Lined Circular Duct Containing Uniform Mean Flow. In 11th AIAA/CEAS Aeroacoustics Conference, number May, pages 23-25, Reston, Virigina, may 2005. American Institute of Aeronautics and Astronautics. ISBN 978-1-62410-052-9. doi: 10.2514/6.2005-3020. URL http://arc.aiaa.org/doi/10.2514/6.2005-3020.

[16] N. Peake and W. Boyd. Approximate Method for the Prediction of Propeller Noise Nearfield Effects. Journal of Aircraft, 30(5), 1993.

[17] A. McAlpine, M.J. Fisher, and B.J. Tester. Buzz-saw noise: A comparison of modal measurements with an improved prediction method. Journal of Sound and Vibration, 306:419-443, 2007.

[18] M. Carley. Propeller Noise Fields. Journal of Sound and Vibration, 233(2): 255-277, June 2000. ISSN 0022460X. doi: 10.1006/jsvi.1999.2797. URL http://linkinghub.elsevier.com/retrieve/pii/S0022460X99927979.

[19] P. R. Prentice. Energy transport in rotating sound fields. Proceedings: Mathematical and Physical Sciences, 441:83-96, 1993. 
[20] T. Node-Langlois, F. Wlassow, V. Languille, Y. Colin, B. Caruelle, J. Gill, X. Chen, X. Zhang, and A. Parry. Prediction of Contra-Rotating Open Rotor broadband noise in isolated and installed configurations. In 20th AIAA/CEAS Aeroacoustics Conference, 2014. 